ARTICLE

\title{
Paradoxical activation of the protein kinase- transcription factor ERK5 by ERK5 kinase inhibitors
}

\author{
Pamela A. Lochhead (10 ${ }^{1 凶}$, Julie A. Tucker (10 2 ${ }^{2}$, Natalie J. Tatum (10 ${ }^{3}$, Jinhua Wang (1) 4,5, David Oxley", \\ Andrew M. Kidger (1) ${ }^{1}$, Victoria P. Johnson (1) 1,6, Megan A. Cassidy ${ }^{1}$, Nathanael S. Gray ${ }^{4,5}$, \\ Martin E.M. Noble (i) ${ }^{3} \&$ Simon J. Cook (iD ${ }^{1 \times}$
}

The dual protein kinase-transcription factor, ERK5, is an emerging drug target in cancer and inflammation, and small-molecule ERK5 kinase inhibitors have been developed. However, selective ERK5 kinase inhibitors fail to recapitulate ERK5 genetic ablation phenotypes, suggesting kinase-independent functions for ERK5. Here we show that ERK5 kinase inhibitors cause paradoxical activation of ERK5 transcriptional activity mediated through its unique C-terminal transcriptional activation domain (TAD). Using the ERK5 kinase inhibitor, Compound 26 (ERK5-IN-1), as a paradigm, we have developed kinase-active, drug-resistant mutants of ERK5. With these mutants, we show that induction of ERK 5 transcriptional activity requires direct binding of the inhibitor to the kinase domain. This in turn promotes conformational changes in the kinase domain that result in nuclear translocation of ERK5 and stimulation of gene transcription. This shows that both the ERK5 kinase and TAD must be considered when assessing the role of ERK 5 and the effectiveness of anti-ERK5 therapeutics.

\footnotetext{
${ }^{1}$ Signalling Laboratory, The Babraham Institute, Babraham Research Campus, Cambridge CB22 3AT, UK. ${ }^{2}$ York Biomedical Research Institute and Department of Biology, University of York, York Y010 5DD, UK. ${ }^{3}$ CRUK Newcastle Drug Discovery Unit, Newcastle University Centre for Cancer, Newcastle University, Newcastle NE2 4HH, UK. ${ }^{4}$ Department of Cancer Biology, Dana-Farber Cancer Institute, Boston, MA 02215, USA. ${ }^{5}$ Department of Biological Chemistry and Molecular Pharmacology, Harvard Medical School, Boston, MA 02115, USA. ${ }^{6}$ Present address: Institute of Cancer Research, Chester Beatty Laboratories, 237 Fulham Road, London SW3 6JB, UK. 『email: pamela.lochhead@babraham.ac.uk; simon.cook@babraham.ac.uk
} 
xtracellular signal-regulated kinase 5 (ERK5, also known as - Big MAP Kinase or BMK1) $)^{1,2}$ is a member of the mitogenactivated protein kinase (MAPK) family, which also includes ERK $1 / 2^{3}$, the JNKs ${ }^{4}$ and the p38 kinases ${ }^{5}$. Like ERK1/2, ERK5 is the effector kinase of a three-tiered MAPK pathway, comprising MEKK2 and MEKK3 (the MKKKs), MEK5 (MKK) and finally ERK5 (MAPK). ERK5 is encoded by the MAPK7 gene and includes an N-terminal kinase domain that shares $50 \%$ identity with ERK2 ${ }^{1,2}$. However, it also contains a large, unique C-terminal extension that includes a nuclear localisation signal (NLS) and a transcriptional activation domain (TAD) (Fig. 1a) ${ }^{6}$. The ERK5 pathway is activated by mitogens ${ }^{7}$, agonists of the Tolllike receptor- $2^{8}$ and cellular stresses ${ }^{9}$. Upon cellular stimulation, activated MEK5 phosphorylates the TEY motif in the ERK5 activation-loop, leading to activation of its kinase domain ${ }^{10}$. The ERK5 C-terminus also becomes auto-phosphorylated and promotes ERK5 translocation from the cytosol to the nucleus ${ }^{11,12}$, where ERK5 has been shown to interact with MEF2 transcription factors such as MEF2D $7,13,14$. The C-terminus can also be regulated by other protein kinases, including ERK1/2 15 and CDK1 $1^{16,17}$, which phosphorylate C-terminal residues independently of ERK5 kinase activity. Thus, the C-terminus mediates some of the effects of ERK5 kinase activity and integrates signals from other pathways.

There is a growing appreciation of the role that ERK5 signalling plays in some diseases, most notably in inflammation and cancer. For example, ERK5 plays a pro-inflammatory role in human endothelial cells and monocytes ${ }^{8,18}$ and ERK5 inhibition exerts an anti-inflammatory effect. ERK5 is also implicated as a mediator of inflammation-driven cancer ${ }^{19,20}$. Finally, a range of studies have suggested that ERK5 signalling promotes cell proliferation, cell survival and motility and invasion $^{21-23}$. While MEK5 or ERK5 mutations are rare in cancer, these components are sometimes over-expressed; indeed, MAPK7 is amplified in hepatocellular carcinoma (HCC) ${ }^{24}$, although it appears not to drive HCC cell proliferation ${ }^{25}$. In addition, ERK5 is activated in melanoma cells with BRAF mutations ${ }^{26}$ and such cells can acquire resistance to the BRAF inhibitor vemurafenib by increasing ERK5 phosphorylation ${ }^{27}$. Thus, ERK5 may drive key cancer hallmarks and promote resistance to other targeted agents.

These observations have prompted commercial and academic MEK5 or ERK5 drug discovery programmes in the hope of developing novel anti-inflammatory or anti-cancer therapeutics. Commercial MEK5 or ERK5 inhibitor programmes include

a

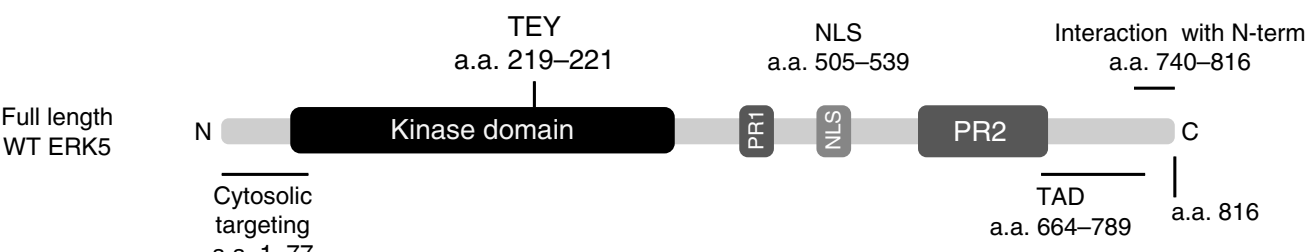

ERK5 $\triangle \mathrm{TAD}$

$\mathrm{N}$ Kinase domain<smiles>C1CCCCC1</smiles>

a.a. 492

b

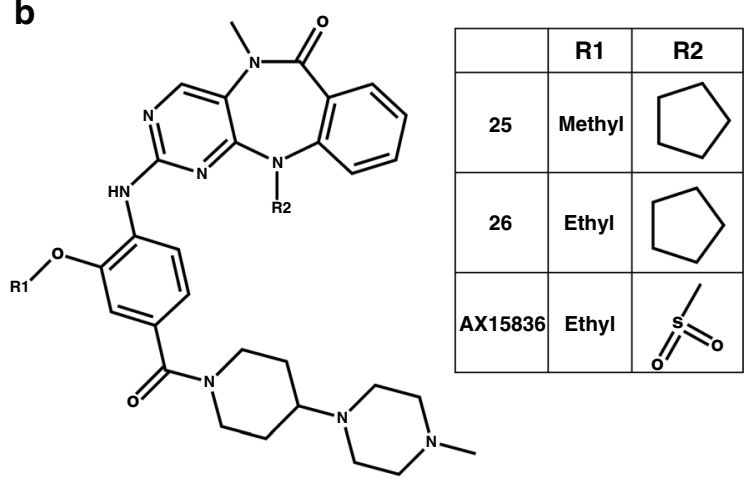

d<smiles>CN(C)Cc1cccc(NC(=C2C(=O)Nc3cc(C(=O)N(C)C)ccc32)c2ccccc2)c1</smiles>

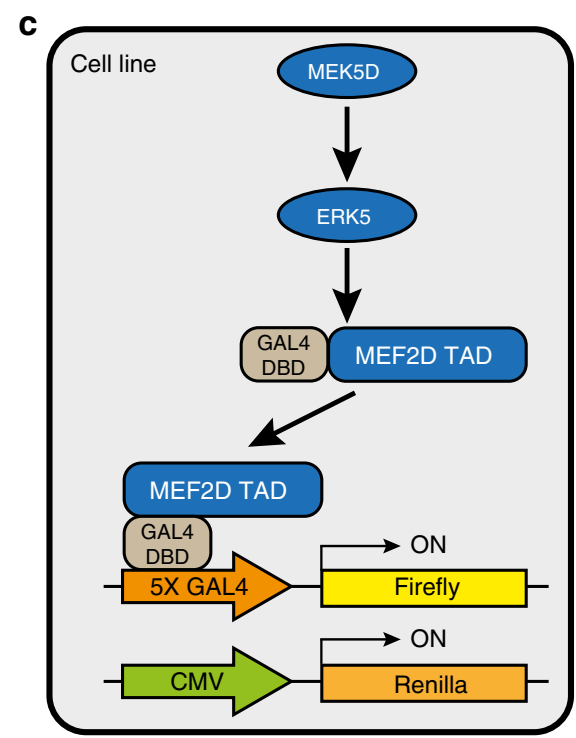

Fig. 1 Schematic diagrams of the reagents used in this study. a Schematic diagram of ERK5 (full length) and ERK5 $\triangle T A D$, which lacks the C-terminal extension. Functional domains with amino acid positions are: cytosolic targeting domain (1-77), kinase domain (48-383), activation-loop TEY motif (219-221), proline rich domain (PR) 1 (434-485) and 2 (578-701), nuclear localisation signal (NLS) (505-539), minimal transactivation domain (TAD) (664-789), and the N-terminal interaction domain (740-816). b Chemical structures of ERK5i: compounds 25, 26 and AX15836. c Schematic representation of the ERK5:MEF2D luciferase assay. $\mathbf{d}$ Chemical structure of the MEK5i, BIX02189. Structures were drawn using ChemDraw v16.0. 
ActivX, Kyorin Pharmaceutical Co. ${ }^{28}$, Bayer $\mathrm{AG}^{29}$, Boehringer Ingelheim $^{30}$ and AstraZeneca ${ }^{31}$. The first ERK5 inhibitor (ERK5i) to be described was XMD8-92 from the Dana-Farber Cancer Institute and the Scripps Research Institute, USA ${ }^{32,33}$. Using the KiNativ method ${ }^{34,35}$ XMD8-92 was found to inhibit ERK5 with an $\mathrm{IC}_{50}$ of $1.5 \mu \mathrm{M}$, being tenfold more selective than its most potent off-target kinases. In cells both siRNA knockdown of ERK5 or XMD8-92 treatment caused an increase in p2 ${ }^{\mathrm{CIP} 1}$ geneexpression, and XMD8-92 or expression of a dominant-negative ERK5 (AEF: where the activation-loop TEY phosphorylation sites are mutated to AEF) decreased tumour growth ${ }^{32,33}$. The subsequent ERK5 inhibitors, compounds 25 and 26 (Fig. 1b), were generated through a collaboration between the Dana-Farber Cancer Institute, USA, and the Structural Genomics Consortium in Oxford, UK and others. Compounds 25 and 26 (also known as ERK5-IN-1 and XMD17-109) are more potent than XMD8-92, and compound 26 represents a further improvement in selectivity over XMD8-92 36,37. Recently, AX15836 (Fig. 1b), a new, more potent and more selective ERK5 inhibitor was developed at ActivX Biosciences Inc, USA and Kyorin Pharmaceutical Co., Japan $^{28}$. Notably, AX15836 did not share with XMD8-92 the ability to block immune responses or inhibit tumour cell proliferation. This led to the discovery that XMD8-92 derived most of its biological activity from off-target interactions with bromodomain-containing proteins such as BRD4. Thus, dual ERK5-BRD inhibitors, such as XMD8-92 and compound 26, are no longer suitable for dissecting the contribution that ERK5 plays within the cell. Selective ERK5 inhibition, without activity against BRD proteins, can be achieved with AX15836 or the new ERK5i compound $46^{38}$ making them useful small molecules for interrogating the cellular roles of ERK5. Critically, AX15836 failed to replicate the effects of genetic ablation of ERK5 on cytokine release from immune cells or cell proliferation ${ }^{28}$. The authors suggested that this disconnect between ERK5 kinase inhibition and ablation of ERK5 expression was evidence of kinaseindependent effects of ERK5 signalling ${ }^{28}$.

Here, we provide an important new insight into this by showing that a variety of ERK5 inhibitors, including compounds 25 and 26 but also AX15836, while being very effective at inhibiting the ERK5 kinase domain cause a paradoxical activation of the ERK5 transcriptional activation domain and drive ERK5dependent gene transcription. Critically our study suggests that both the ERK5 kinase domain and the ERK5 TAD must be considered when validating the role of ERK5 in biological processes, in diseases where ERK5 is implicated and in designing and assessing the effectiveness of anti-ERK5 therapeutics.

\section{Results}

ERK5i induce transcriptional activity in the ERK5:MEF2D reporter system independently of kinase activity. The paucity of well-validated ERK5 substrates led us to use an ERK5-dependent transient transfection reporter assay to determine the 'in cell' $\mathrm{IC}_{50}$ of small-molecule ERK5 kinase inhibitors (ERK5i). This system (Fig. 1c) comprised: (i) a constitutively active form of the ERK5 kinase MEK5, MEK5D (where the regulatory activation-loop phosphorylation sites in MEK5 are mutated to phospho-mimetic aspartic acid, producing a constitutively active form of MEK5), to phosphorylate and activate ERK5; (ii) HA-tagged, full length human ERK5; (iii) a fusion protein of the transactivation domain (TAD) of MEF2D and the DNA binding domain of the yeast transcription factor GAL4 (MEF2D is one of the few validated ERK5 substrates and interacting proteins ${ }^{6,7,14,39}$ ); (iv) a luciferase reporter construct with upstream multimerised GAL4 binding sites and (v) a Renilla reporter construct that is regulated by the constitutive CMV promoter as a transfection control. In this system, MEK5D phosphorylates the activation-loop of ERK5 causing activation of its kinase domain. The kinase domain of ERK5 then phosphorylates the MEF2D TAD leading to activation, thereby driving luciferase expression, which serves as a readout of ERK5 kinase activity. We previously validated this system and used it to monitor ERK5 activity ${ }^{25,40,41}$. The ERK5 inhibitors compound 26, compound 2532,37, AX15836 28 (Fig. 1b) and MEK5 inhibitor BIX02189 ${ }^{30}$ (Fig. 1d) were used in our studies.

To measure the $\mathrm{IC}_{50}$ of ERK5i, transfected cells (as described above (Fig. 1c)) were incubated with increasing concentrations of inhibitors and luciferase activity measured. Compound 26 caused a dose-dependent decrease in MEK5D-driven luciferase activity; however the dose-response curve never reached zero, with $30-40 \%$ of maximal luciferase activity persisting, even at higher concentrations (Fig. 2a). This effect was also seen with compound 25 (an ERK5i similar to compound 26) (Fig. 2b). Compound 26 also inhibits the bromodomain and extra terminal (BET) domain family member, BRD4, a protein that binds acetylated lysines and plays a role in gene regulation. To control for BRD4 being responsible for the residual activity in the ERK5:MEF2D assay we used AX15836 an ERK5i that has no BRD4 activity ${ }^{28}$. Strikingly AX15836 caused a paradoxical increase in ERK5:MEF2Ddependent luciferase expression over and above that seen with MEK5D (Fig. 2c).

We speculated that the residual activity that remained for compounds $\mathbf{2 5}$ and 26, and the increase in activity with AX15836 was due to an ERK5-mediated induction of MEF2D activity in response to ERK5i binding. To test if ERK5i were able to drive ERK5:MEF2D activity in this assay we repeated these experiments but omitted MEK5D, so that ERK5 was un-phosphorylated and inactive ('non-activated full length ERK5'). Indeed, compounds 26, 25 and AX15836 all elicited a dose-dependent increase in ERK5: MEF2D luciferase activity. Moreover, for compounds 26 and 25 the level of induced activity was the same as the residual activity seen when MEK5D was present (Fig. 2a-c comparing 'non-activated full length ERK5' with 'MEK5D-activated full length ERK5').

The C-terminus of ERK5 contains a transcriptional transactivation domain $(\mathrm{TAD})^{6}$ (Fig. 1a), which is known to interact with MEF2D ${ }^{6}$. To test if the C-terminus was responsible for the residual activity we produced a mutant of ERK5 (termed ERK5 $\triangle \mathrm{TAD}$ ) that lacked the C-terminal extension, mimicking a naturally occurring ERK5 splice variant ${ }^{42}$ (Fig. 1a). MEK5D activated both full length ERK5 and ERK5 $\triangle$ TAD equally in this system (Fig. 2d). Using MEK5D to activate ERK5 $\triangle$ TAD we saw complete, saturable inhibition of ERK5:MEF2D-dependent luciferase activity with compounds 25, 26 and AX15836 (Fig. 2a-c) consistent with their ERK5 kinase inhibitory activity. In agreement with the paradoxical activation of ERK5 being mediated by its C-terminal extension, there was no induction of luciferase activity when non-activated ERK5 $\triangle \mathrm{TAD}$ (not activated by MEK5D) was incubated with either compounds 25, 26 or AX15836 (Fig. 2a-c). Furthermore, using successive C-terminal deletion mutants we mapped the region responsible for compound 26-induced transcriptional activation of ERK5: MEF2D to the previously reported minimal TAD (for more information see Supplementary Fig. 1).

The activation of ERK5:MEF2D seen with ERK5i was not seen when we used the MEK5 inhibitor, BIX02189; when tested with full length ERK5, BIX02189 fully inhibited ERK5:MEF2D luciferase activity with an $\mathrm{IC}_{50}$ of $\sim 500 \mathrm{nM}$ (Fig. 2e). To test if ERK5 kinase activity was required for paradoxical activation of ERK5 we repeated the same experiments with a kinase-dead mutant of ERK5 in which the catalytic aspartic acid was mutated to alanine (D182A), (Fig. 2f); both compounds 26 and AX15836 stimulated ERK5-dependent expression of luciferase 

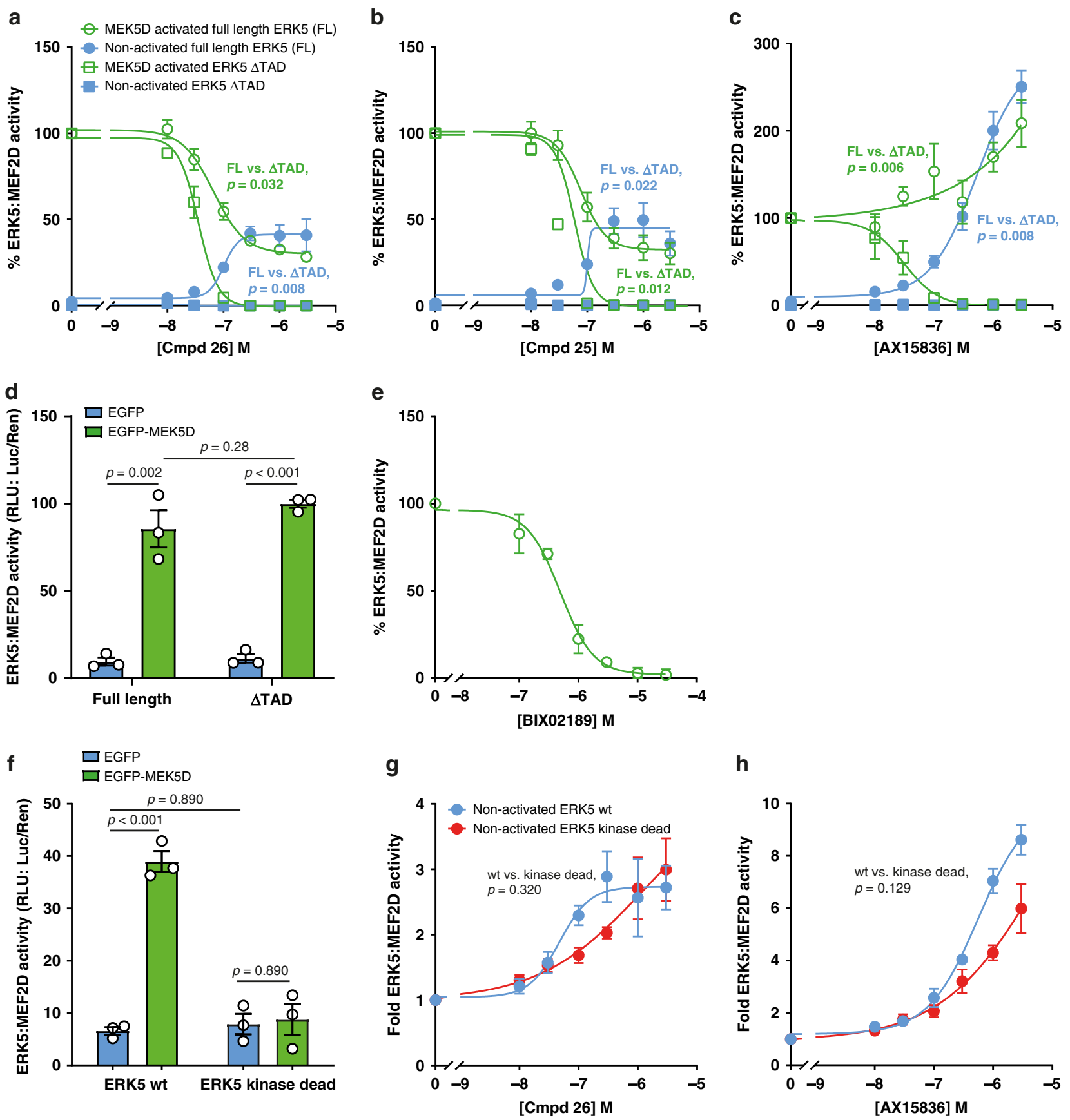

Fig. 2 ERK5i induce transcriptional activity in the ERK5:MEF2D reporter system independently of kinase activity. a, b, c and e HEK293 cells were transfected with GAL4-MEF2D, GAL4:LUC and CMV:Renilla, together with either wild-type HA-ERK5 (full length) or HA-ERK5 $\triangle$ TAD and EGFP-MEK5D or EGFP (control) as indicated. Four hours post transfection, cells were treated with either DMSO (control) or compound 26 (a), compound $\mathbf{2 5}$ (b), AX15836 (c) or BIX02189 (e) at concentrations indicated. Twenty-four post transfection, cells were lysed and firefly luciferase activity was measured and normalised to Renilla. The results are presented as the mean of three independent experiments \pm SEM. Source data are provided as a Source Data file. $\mathbf{d}$ HEK293 cells were transfected as in (a). Twenty-four hours post transfection cells were lysed and processed as in a. $\mathbf{f} H E K 293$ cells were transfected with GAL4-MEF2D, GAL4:LUC and CMV:Renilla together with either wild-type HA-ERK5 (full length) or HA-ERK5 kinase dead and EGFP-MEK5D or EGFP (control) as indicated. Twenty-four hours post transfection cells were lysed and processed as in $\mathbf{a} . \mathbf{g}$ and $\mathbf{h}$ HEK293 cells were transfected as in (f). Four hours post transfection, cells were treated with either DMSO (control) or compound $\mathbf{2 6}$ (g) or AX15836 (h) at concentrations indicated. Twenty-four hours post transfection cells were lysed and processed as in (a).

by MEF2D with either wild type or kinase dead ERK5 (Fig. 2g, h). Phosphorylation of the ERK5 C-terminus and MEF2D promotes transcriptional activity. We therefore assessed C-terminal phosphorylation and MEF2D phosphorylation in the presence of MEK5D, compound 26 and AX15836. Although compounds 26 and AX15836 promoted phosphorylation of S754 in the ERK5
C-terminus, this was not required for ERK5i induced ERK5: MEF2D activity (Supplementary Fig. 2). Furthermore, we identified 18 MEK5-ERK5-driven phosphorylation sites on MEF2D, but phosphorylation of MEF2D was not induced by compound $\mathbf{2 6}$ or AX15836 (for more information see Supplementary Fig. 3 and Supplementary Table 1). 
Taken together these results demonstrated that while compounds 25, 26 or AX15836 are all very effective inhibitors of ERK5 kinase activity, they also promote paradoxical activation of the ERK5 C-terminal TAD. This effect does not require ERK5 kinase activity, ERK5 or MEF2D phosphorylation and is not shared with MEK5i (BIX02189), suggesting it is unique to those compounds that bind to ERK5.

Generation of compound 26-resistant, kinase-active mutants of ERK5. In cell-based assays, compounds may exhibit off-target effects, which create false positives. These include: (i) activation of stress responses that may signal to ERK5; (ii) indirect effects on luciferase expression by affecting the transcriptional and translational machinery, or (iii) direct effects on luciferase activity. Therefore, it was critical to determine whether the transcriptional activation by ERK5i in the ERK5:MEF2D assay was a result of direct binding of ERK5i to the ERK5 kinase domain and not an off-target effect. We, therefore, set out to generate ERK5 mutants that lacked the ability to bind compound $\mathbf{2 6}$ but retained kinase activity.

The X-ray crystal structure of the kinase domain of ERK5 in complex with compound $25^{37}$ and pan-kinase binding selectivity data for the highly similar compound $26^{36}$ suggested that the presence of a small residue immediately prior to the DFG motif, at the start of the activation-loop (G199 in ERK5), combined with a leucine in the base of the ATP-binding pocket (L189 in ERK5), was important to accommodate the cyclopentyl substituent of compounds 25 and 26 (Fig. 3a, b). This combination of residues, although common among kinases more generally, is rare in members of the MAPK family, being found only in ERK5 (Fig. 3c). ERK1, ERK2, ERK7 and NLK, all have a cysteine at the position equivalent to ERK5 G199 combined with a leucine at the position equivalent to ERK5 L189, and these kinases are not inhibited by compound 26. By contrast, ERK3 and ERK4, and the polo-like kinases PLK1, PLK2 and PLK3, which are also not inhibited by compound $\mathbf{2 6}$, all have glycine at the position equivalent to G199 in ERK5, while the residue equivalent to ERK5 L189 is the bulkier phenylalanine. We hypothesised therefore that introducing individual mutations in the ATPbinding site of ERK5 of L189 to phenylalanine (L189F) and G199 to cysteine (G199C) would generate mutant ERK5 variants resistant to inhibition by compound $\mathbf{2 6}$, while retaining kinase activity. Elkins et al. ${ }^{37}$ postulate that binding affinity and selectivity are also driven by hydrophobic interaction of I115 at the back of the ATP-binding pocket with the $N$-methyl substituent of compounds 25 and 26. Comparison of binding data and sequence conservation across the family of DCLKs suggested that replacement of I115 with valine (as observed in DCLK1 and 3) would negatively impact binding of compounds 25 and 26 (Fig. 3a-c), again generating a kinase-active but inhibitor-resistant mutant. Finally, the presence of aspartate (D143 in ERK5) at the mouth of the ATP-binding pocket just Cterminal to the hinge region is proposed to contribute to tight binding of compounds $\mathbf{2 5}$ and $\mathbf{2 6}$ by securing the hinge-binding moiety in place ${ }^{37}$ (Fig. 3a, b). The side-chain of D143 forms a helix cap, stabilising the short $\alpha \mathrm{D}$ helix, which follows the hinge region (Supplementary Fig. 4A). Structure-guided sequence alignments across the kinome show that this residue is often an amino acid such as aspartate, asparagine, glutamate or serine, which can form a helix cap (Supplementary Fig. 4B) ${ }^{43}$; truncation to glycine (D143G) removes both the steric constraints imposed on compound binding and the $\mathrm{aD}$ helix cap.

We undertook molecular dynamics simulations of compound 26 bound to wild-type ERK5, as well as mutants I115V, D143G, L189F and G199C to predict relative binding energies by the
MM/PBSA method (Fig. 3d). We noted no significant changes in the flexibility of either the ligand or the binding site (Supplementary Fig. S4C, D) over the short simulation time; however, as anticipated, we observed unfavourable changes to the binding energies of all mutants, with statistically significant reductions for mutants I115V and L189F compared to wild-type. Converting the differences in binding energy to estimate a log-fold change in binding affinity (Fig. 3e), predicted notably weaker binding of compound $\mathbf{2 6}$ to all the ERK5 mutants simulated, with the binding position of compound $\mathbf{2 6}$ compromised by all mutations (Fig. 3f-i).

We generated these mutants and expressed them in the presence and absence of MEK5D. MEK5D was able to phosphorylate all of the mutants on the TEY motif, albeit D143G to a lesser extent than the others (Fig. 3j). An ERK5 band-shift was observed for wild type, I115V, L189F and G199C but not kinase dead or D143G, suggesting that D143G lacks kinase activity. Consistent with this, phosphorylation of T733, a known autophosphorylation site was detected on wild type, I115V, L189F and G199C but not kinase dead or D143G. Furthermore, S754 phosphorylation (another ERK5 autophosphorylation site) was increased following MEK5D expression on wild type, I115V, L189F and G199C, but not kinase dead or D143G. Interestingly, G199C had higher basal S754 phosphorylation than wild type (Fig. 3j). As we saw a MEF2D band-shift when ERK5 was activated with MEK5D in Supplementary Fig. 3C, we used this as a readout of ERK5 kinase activity in cells; this demonstrated that wild type, I115V, L189F and G199C were all active MEF2D kinases, whereas D143G was not (Fig. 3j). Next, we assessed the activity of these mutant forms of ERK5 in the ERK5:MEF2D luciferase assay; L189F and G199C had a higher basal activity compared to wild type and $1115 \mathrm{~V}$, L189F and G199C were all activated by MEK5D, whereas D143G was not (Fig. 3k). Thus, we were able to generate three kinase-active ERK5 mutants with predicted reduced binding ability for compound $\mathbf{2 6 .}$

Binding of compounds 26 or AX15836 to the ERK5 kinase domain is required for paradoxical ERK5:MEF2D transcriptional activation. We conducted dose-response experiments comparing the predicted compound 26-resistant ERK5 mutants with wild-type ERK5 in the ERK5:MEF2D assay. With nonactivated ERK5, L189F and G199C were both completely resistant to compound 26 with no induction of ERK5:MEF2D (Fig. 4a, b), while I115V and D143G had reduced sensitivity to compound 26 compared to wild type so that the induction curve shifted to the right (Fig. 4c, d). For MEK5D-activated ERK5 we assessed the effects on L189F, G199C and I115V, but not D143G (as it could not be activated). L189F, G199C and I115V ERK5 mutants each showed a reduction in sensitivity to compound $\mathbf{2 6}$, with the effect more notable for L189F and G199C compared to I115V (Fig. $4 \mathrm{e}-\mathrm{g}$ ). This is consistent with the induction of non-activated ERK5:MEF2D transcriptional activity for these mutants.

AX15836 is a derivative of XMD8-92 and compound 26, so we speculated that the compound 26 drug-binding mutants would also be resistant to AX15836. We therefore repeated the dose-response experiments comparing the compound 26-resistant ERK5 mutants to wild type in the ERK5:MEF2D assay. With non-activated ERK5, L189F and G199C were both completely resistant to AX15836 (Fig. 4h-i) and I115V and D143G had a reduced effect (Fig. 4j-k), comparable to compound 26.

These results confirmed the structural predictions that $1115 \mathrm{~V}$, D143G, L189F and G199C would reduce binding of compound 26 to ERK5. These mutants fell into two groups; those on which compound 26 had little or no effect (L189F and G199C), and 
a

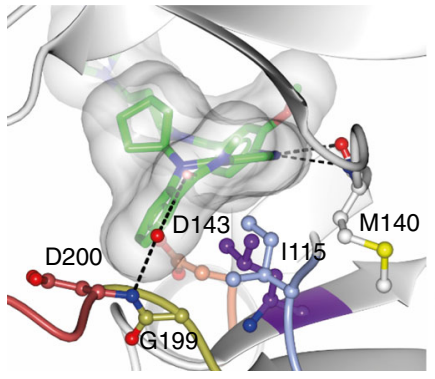

d

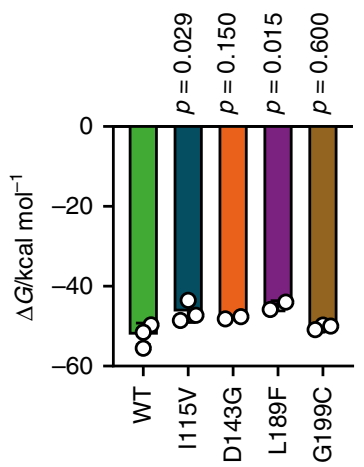

e

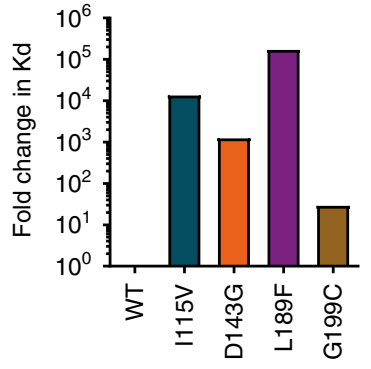

b

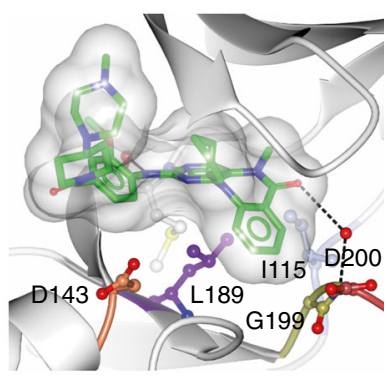

C

DCLK1 447 NIVLL DCLK2 $451 \mathrm{NI}$ I ML DCLK3 413 NIVKL ERK1 $99 \mathrm{NVI} \mathrm{GI}$ ERK2 $82 \mathrm{NI} \mid \mathrm{G}$ I ERK3 76 NIVKV ERK4 76 NIVKV ERK5 $113 \mathrm{NI} \mid \mathrm{AI}$ ERK6 85 NVIGL ERK7 $72 \mathrm{NI}$ I SL PLK1 112 HVVGF PLK2 $141 \mathrm{HVV} \mathrm{QF}$ PLK3 $121 \mathrm{HIVRF}$ PLK4 71 S닡느
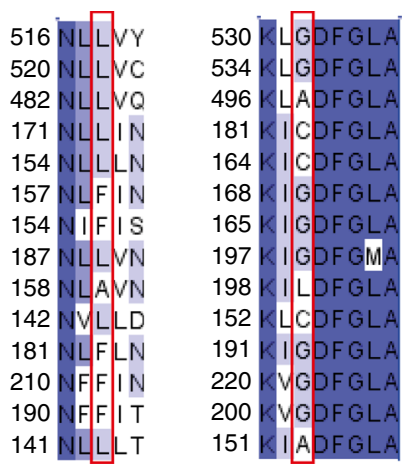

j

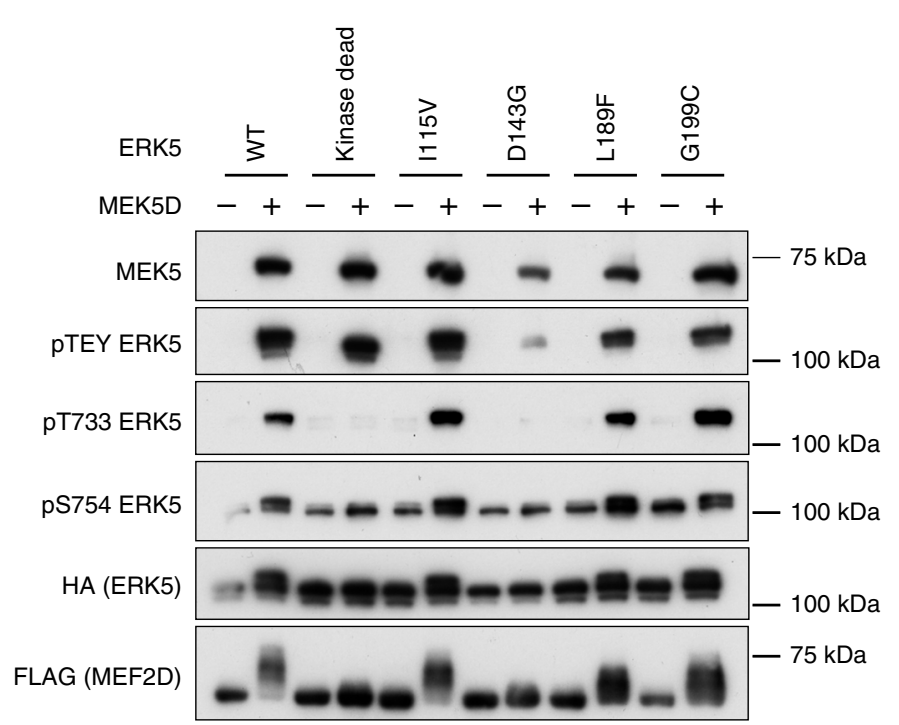

f $\quad$ g

g

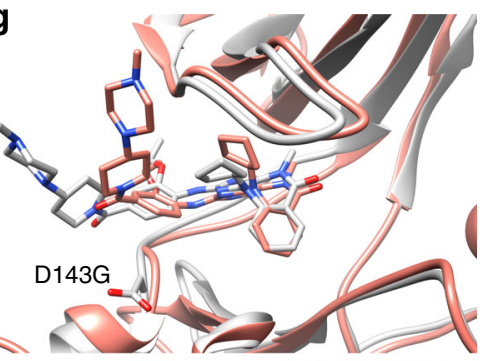

h

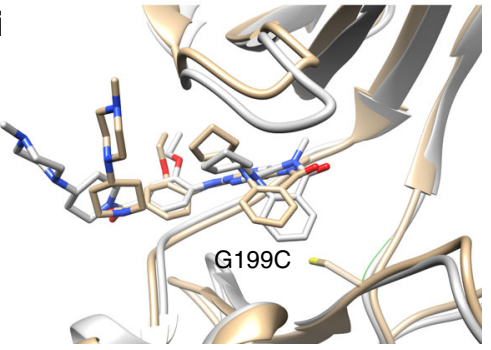

$\mathbf{k}$

口EGFP
口EGFP-MEK5D

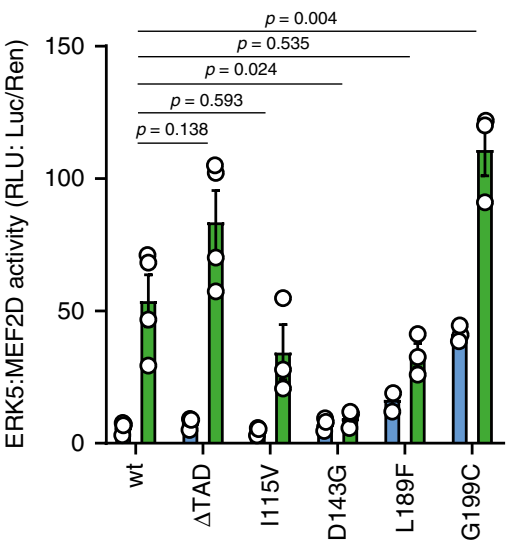

those on which compound $\mathbf{2 6}$ had a reduced effect (I115V and D143G). These mutants exhibited similar responses to AX15836. Significantly, these results showed that direct binding of compound 26 or AX15836 to the ERK5 kinase domain was required for the paradoxical drug-induced activation of ERK5: MEF2D transcriptional activity, confirming that compound $\mathbf{2 6}$ or AX15836 were acting 'on target' to activate the ERK5 TAD.
Compound 26 and AX15836 promote ERK5 nuclear localisation. In its inactive state, ERK5 is predominantly cytosolic and translocates into the nucleus upon activation by MEK5 $5^{12}$. This is regulated by an intramolecular interaction between the $\mathrm{N}$ terminal kinase domain and the C-terminus, which ensures mutual repression of the kinase and TAD functions; this is derepressed by MEK5-catalysed phosphorylation of ERK5, which 
Fig. 3 Generation of compound 26-resistant kinase-active mutants of ERK5. a, b Compound $\mathbf{2 5}$ (carbon atoms in green) bound to the ATP-binding pocket of ERK5 (PDB 4B9937). Side-chains of residues proposed by Elkins et al. ${ }^{37}$ as key to the selectivity and binding affinity of $\mathbf{2 5}$ for ERK5 are shown in ball-and-stick and coloured blue (Ile115), purple (Leu189), gold (Gly199) and coral (Asp143), respectively. Asp200 from the 'DFG' motif at the start of the activation-loop (red), and Met140 in the hinge region (grey) are also shown. A transparent molecular surface is drawn over compound $\mathbf{2 5}$. Hydrogen bonds between $\mathbf{2 5}$ and ERK5 are shown as black dashed lines. The view in (b) is rotated $\sim 90^{\circ}$ about a vertical axis compared to the view in (a). Figure prepared using CCP4MG 84 . c Comparison of sequences across the DCLK, ERK and PLK families highlights conservation of Ile115, Leu189 and Gly199 (ERK5 numbering), respectively, as key to inhibition by compounds $\mathbf{2 5}$ and $\mathbf{2 6}$. Sequences were retrieved from the UniProt database ${ }^{85}$, aligned using Clustal Omega ${ }^{86}$, and the alignment coloured by sequence identity (where darker blue indicates higher conservation) and annotated using Jalview ${ }^{87}$. $\mathbf{d}$ Calculated binding energies for compound $\mathbf{2 6}$ to wild-type ERK5, and the mutant variants I115V, D143G, L189F and G199C, as derived from molecular dynamics simulations and MM/PBSA calculation (unadjusted for entropic contribution); the reduction in binding energy is statistically significant by one-way ANOVA for binding of compound $\mathbf{2 6}$ to mutants I115V ( $p=0.0282)$ and L189F $(p=0.0149)$ when compared to binding to wild-type ERK5. Source data are provided as a Source Data file. e Estimated log-fold change in Kd derived from calculated binding energies demonstrating notably weaker affinity of compound $\mathbf{2 6}$ for all mutant variants of ERK5 compared to wild-type. Source data are provided as a Source Data file. $\mathbf{f}-\mathbf{i}$ Comparison of compound $\mathbf{2 6}$ binding to ERK5 wild-type (grey) and I115V (f, blue), D143G (g, coral), L189F (h, purple) or G199C (i, gold) in silico, derived from molecular dynamics simulations with wild-type and mutated residue shown as sticks to denote mutation site. The D143G mutation causes compound $\mathbf{2 6}$ to shift toward the Ploop. The I115V, L189F and G199C mutations cause compound $\mathbf{2 6}$ to shift out into a shallower binding position in the active site, resulting in the weaker binding energies observed. Figures were prepared using UCSF Chimera. j HEK293 cells were transfected with FLAG-MEF2D and either wild-type HA-ERK5, kinase dead HA-ERK5, HA-I115V ERK5, HA-D143G ERK5, HA-L189F ERK5 or HA-G199C ERK5, and either EGFP-MEK5D or EGFP. Twenty-four hours post transfection cells were lysed, subjected to SDS-PAGE and immuno-blotted with the antibodies shown. The experiment was repeated three times and a representative image is shown. $\mathbf{k}$ HEK293 cells were transfected with GAL4-MEF2D, GAL4:LUC and CMV:Renilla, together with either wild-type HA-ERK5, HA-ERK5 $\triangle T$ TAD, HA-1115V ERK5, HA-D143G ERK5, HA-L189F ERK5 or HA-G199C ERK5 and either EGFP-MEK5D or EGFP. Twenty-four hours post transfection, cells were lysed and firefly luciferase activity was measured and normalised to Renilla. The results are presented as the mean of at least three independent experiments \pm SEM. Source data are provided as a Source Data file.

activates the kinase domain and exposes the NLS and TAD ${ }^{11,12}$. In order to determine if compound $\mathbf{2 6}$ or AX15836 mimic MEK5D by disrupting this interaction, we co-expressed the HAERK5 $\triangle \mathrm{TAD}$ mutant (i.e., just the kinase domain) with GSTERK5C-term. Pulldown with glutathione Sepharose beads showed that the HA-ERK5 $\triangle$ TAD associated with the GSTERK5C-term; furthermore, expression of MEK5D to drive TEY phosphorylation of HA-ERK5 $\triangle \mathrm{TAD}$ caused it to dissociate from GST-ERK5C-term (Fig. 5b). Compound 26 and AX15836 both caused a dose-dependent dissociation of HA-ERK5 $\triangle \mathrm{TAD}$ from the GST-ERK5C-term despite failing to promote TEY phosphorylation. As a control we used an ERK1/2 inhibitor, SCH772984 ${ }^{44}$, which is inactive towards ERK5. SCH772984 had no effect demonstrating that it was an ERK5i selective effect (Fig. 5a). To show that SCH772984 was active in cells, we expressed myc-BRAFV600E to stimulate ERK1/2 activity and measured phosphorylation of T359 on RSK, an ERK1/2 phosphorylation site. SCH772984 was active as it blocked this phosphorylation (Fig. 5b).

Prompted by this we assessed whether compound $\mathbf{2 6}$ or AX15836 treatment led to the exposure of the NLS to promote ERK5 nuclear translocation. We expressed HA-ERK5 in HeLa cells and imaged HA-ERK5 localisation using fluorescence microscopy. In untreated cells the majority of HA-ERK5 was seen in the cytosol. As a positive control, we co-expressed EGFP-MEK5D, which increased HA-ERK5 nuclear staining. Next, we assessed the effects of compound 26 or AX15836 and also observed an increase in HA-ERK5 nuclear staining (Fig. 5c). We used high-content fluorescence microscopy to score the number of cells with a higher nuclear to cytosolic staining for HA-ERK5. Using this method, we again observed that co-expression of MEK5D increased HA-ERK5 nuclear translocation, as expected. We also demonstrated that compound $\mathbf{2 6}$ or AX15836 promoted HA-ERK5 nuclear translocation in a dose-dependent manner (Fig. 5d, e). An increase in HAERK5 nuclear localisation in response to compound $\mathbf{2 6}$ or AX15836 was seen after $15 \mathrm{~min}$, plateaued at $1 \mathrm{~h}$ (Fig. 5f) and was sustained for up to $24 \mathrm{~h}$ (Fig. $5 \mathrm{~d}$, e). Consistent with these experiments we also saw an increase in endogenous ERK5 in the nucleus following treatment of HeLa cells with compound $\mathbf{2 6}$ or AX15836 (Fig. 5g).
We extended these experiments to determine the cellular localisation of the compound 26-resistant ERK5 mutants and the effects of compound $\mathbf{2 6}$ on these mutants. For the drug-resistant mutants, I115V staining was mostly cytosolic, similar to wild-type ERK5. D143G and L189F exhibited a higher basal nuclear staining than wild type. G199C had an increased nuclear staining with $80 \%$ of cells having more G199C staining in the nucleus than in the cytosol (Fig. 5h). These results reflected the basal ERK5: MEF2D activity measured in Fig. 3k (with the exception of D143G, which had a basal ERK5:MEF2D activity that was comparable to wild-type ERK5). The compound 26-resistant mutants had an attenuated nuclear localisation in response to compound 26 treatment. For I115V the effect of compound 26 was slightly reduced at $0.1 \mu \mathrm{M}$ but exhibited a similar effect to wild type at $1 \mu \mathrm{M}$ (Fig. 5i), as was seen with ERK5:MEF2D activity in Fig. 4c. D143G, L189F and G199C localisation was resistant to compound $\mathbf{2 6}$ treatment (Fig. 5i), consistent with the attenuated effect of compound 26 on the ERK5:MEF2D activity of these mutants shown in Fig. 4.

These results not only showed that compound 26 or AX15836 cause exposure of the NLS and TAD, driving nuclear localisation of ERK5, but also that the level of nuclear localisation of ERK5 correlated with transcriptional activity (comparing the nuclear localisation of the ERK5 compound 26-resistant mutants with basal ERK5:MEF2D activity), and provided further evidence that the compound 26-resistant mutants were less responsive to compound 26.

Compound 26 and AX15836 activate expression from the KLF2 promoter. Finally, we wanted to assess the effects of ERK5i on an ERK5-regulated promoter. To this end, we used KLF2 that is known to be regulated by ERK5 and MEF2D ${ }^{44}$. We expressed a luciferase reporter regulated by $-924 /+14$ bp of the $5^{\prime}$ UTR of the KLF2 gene. Co-expression of EGFP-MEK5D was able to increase KLF2 reporter activity 3.5-fold; acting through endogenous ERK5 and MEF2D. Expression of MEF2D and ERK5 increased KLF2 reporter activity 15-fold in the presence of MEK5D confirming their role in regulating the KLF2 promoter (Fig. 6a). We used this system to measure the effects of compound 26 or AX15836 on endogenous ERK5. Both of these ERK5i increased KLF2 

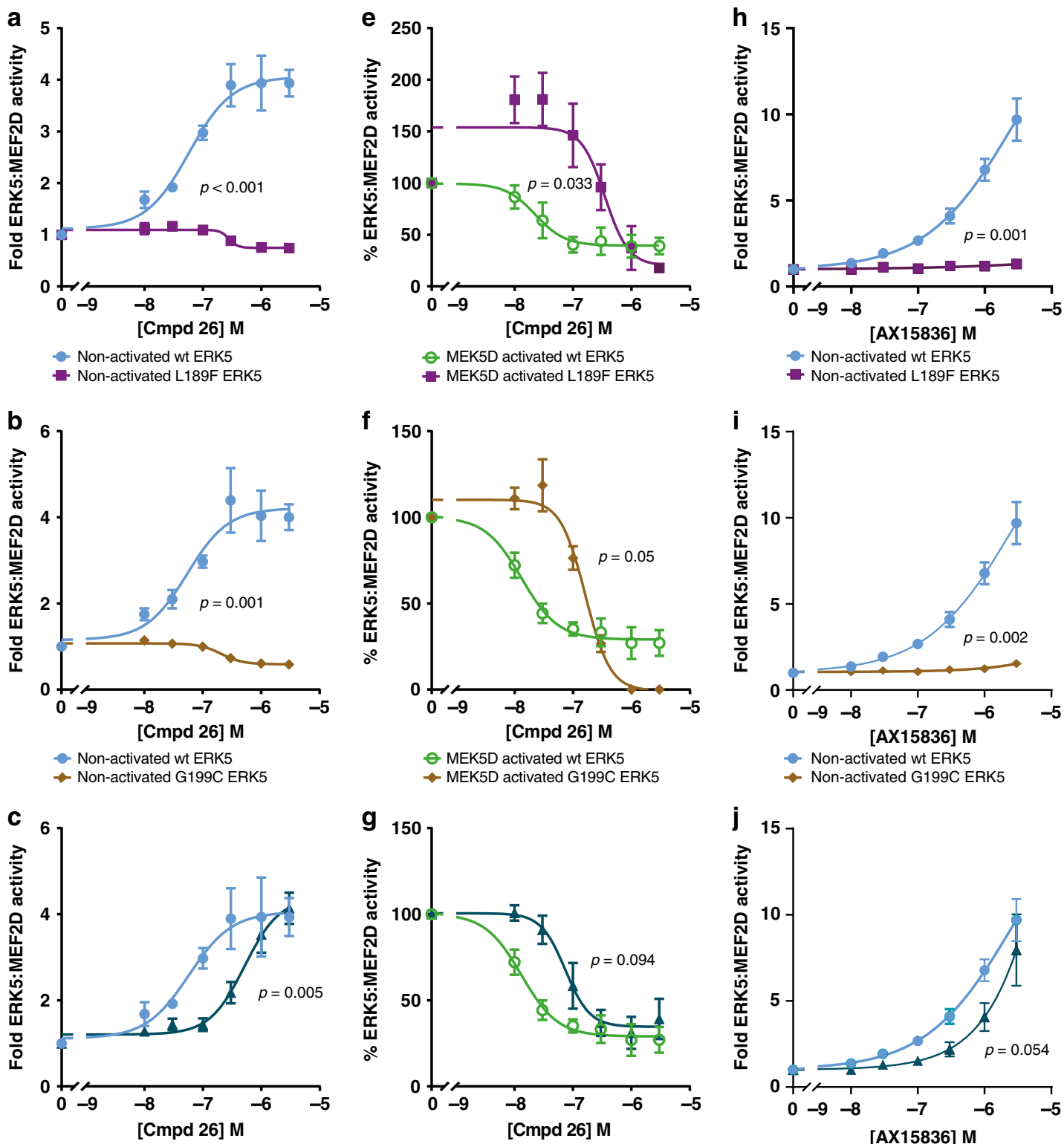

$$
\begin{aligned}
& \ominus \text { MEK5D activated wt ERK5 } \\
& \bullet \text { MEK5D activated G199C ERK5 }
\end{aligned}
$$

$\leadsto$ Non-activated G199C ERK5
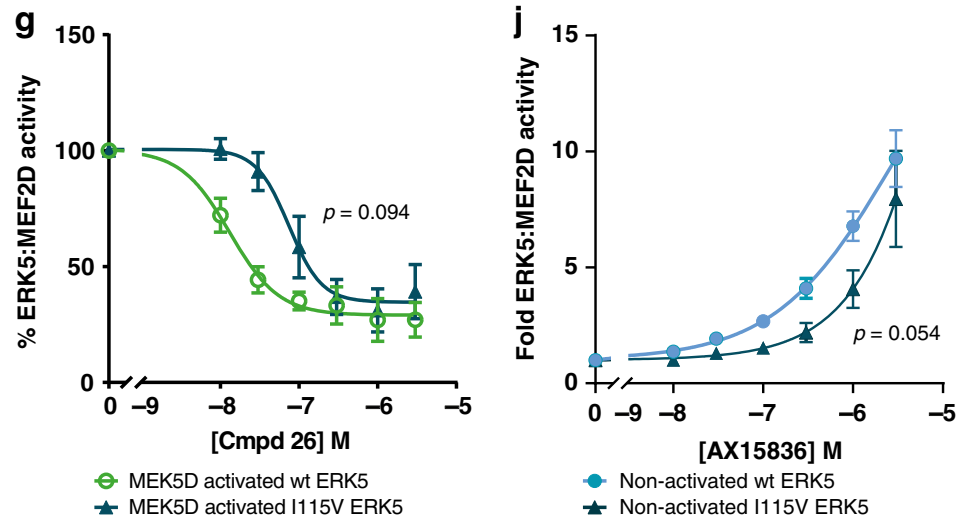

$$
\begin{aligned}
& \text { - Non-activated wt ERK5 } \\
& + \text { Non-activated I115V ERK5 }
\end{aligned}
$$

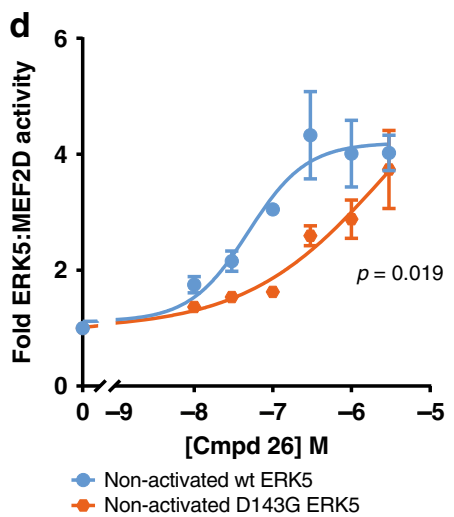

$\theta$ MEK5D activated wt ERK5 士 MEK5D activated I115V ERK5

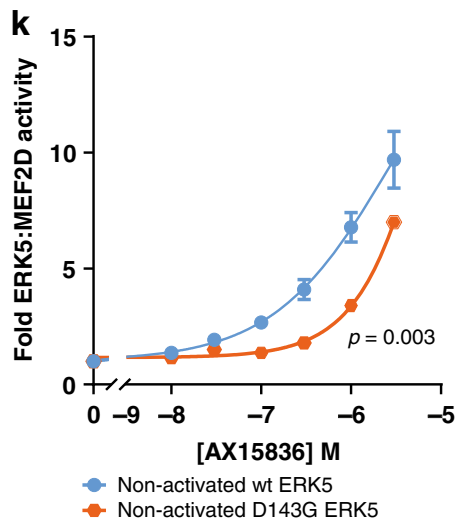

promoter activity in a dose-dependent manner; however, the dose-dependency for each compound was different. The effect of compound 26 peaked at $300 \mathrm{nM}$ before reducing at higher concentrations, whereas the induction observed with AX15836 increased as the concentration of drug increased (Fig. 6b). To test if the bi-phasic effect of compound $\mathbf{2 6}$ was due to the induction of S754 phosphorylation (Supplementary Fig. 2), we repeated this experiment comparing the effects of over-expressed wt ERK5 with the ERK5 S754A mutant, but both wt and the S754A mutant were inhibited at higher concentrations of compound $\mathbf{2 6}$ 
Fig. 4 Binding of compound 26 and AX15836 to the ERK5 kinase domain is required for paradoxical ERK5:MEF2D transcriptional activation. a-d and h-k HEK293 cells were transfected with GAL4-MEF2D, GAL4:LUC and CMV:Renilla together with wild-type HA-ERK5 (full length) and either HA-L189F ERK5 (a, h), HA-G199C ERK5 (b, i), HA-I115V ERK5 (c, j) or HA-D143G ERK5 (d, k) and EGFP then 4 h post transfection treated with compound $\mathbf{2 6}$ (a-d) or AX15836 (h-k) at the concentrations indicated. Twenty-four hours post transfection, cells were lysed and firefly luciferase activity was measured and normalised to Renilla. The results are presented as the mean of three experiments \pm SEM. Source data are provided as a Source Data file. e- $\mathbf{g}$ HEK293 cells were transfected with GAL4-MEF2D, GAL4:LUC and CMV:Renilla together with wild-type HA-ERK5 (full length) and either HA-L189F ERK5 (e), HAG199C ERK5 (f) or HA-I115V ERK5 (g) and EGFP-MEK5D then $4 \mathrm{~h}$ post transfection treated with compound $\mathbf{2 6}$ at the concentrations indicated. Twentyfour hours post transfection, cells were lysed and processed as in (a-d and $\mathbf{h}-\mathbf{k}$ ).

(Supplementary Fig. 5). However, as compound 26 is known to inhibit BRD4, we tested to see if KLF2-luciferase reporter activity was sensitive to BRD4 inhibition using JQ1. At $0.1 \mu \mathrm{M}$ JQ1 KLF2 reporter activity was inhibited by $\sim 30 \%$ (Supplementary Fig. 6). This suggests that at higher concentrations of compound $26(>1 \mu \mathrm{M})$ the reduction in KLF2-luciferase reporter activity may be due to BRD4 inhibition. Notably, AX15836, which does not inhibit BRD4 $4^{28}$, did not cause inhibition at high doses (Fig. 6b).

We then tested the role of the C-terminus of ERK5 using the KLF2 reporter system, with MEK5D as a positive control. Here, $300 \mathrm{nM}$ AX15836 increased full length ERK5 driven KLF2 reporter activity and this was dependent on the C-terminus of ERK5 as the ERK5 $\triangle$ TAD was unable to support KLF2 promoter activity in the presence of AX15836 or MEK5D (Fig. 6c). These results demonstrate that ERK5i are able to drive paradoxical transcriptional activation of a canonical ERK5-regulated gene, as well as the ERK5:MEF2D system.

\section{Discussion}

The identification of ERK5 as a potential anti-cancer and antiinflammatory drug target has seen academic groups and pharmaceutical companies invest in developing small-molecule inhibitors of the ERK5 kinase domain (ERK5i). Kinases are attractive, 'druggable' targets due to their active site lying in a deep cleft between the canonical N- and C-terminal lobes and by 2015, 28 kinase inhibitors had received US FDA approval ${ }^{45}$. However, intensive research and clinical experience have identified two main problems that affect the efficacy of kinase inhibitors: (i) innate or acquired resistance to the kinase inhibitor due to mutation of the intended target ${ }^{46}$, mutation of other pathway components or pathway remodelling ${ }^{47}$ and (ii) unintended activation of the target pathway ${ }^{48}$, either by inhibition of negative feedback pathways ${ }^{49}$ or through inhibitor binding to the kinase resulting in paradoxical activation (the latter termed inhibitor hijacking of kinase activation' by Okuzumi et al. ${ }^{50}$ ).

Our results show that ERK5i bind directly to the ERK5 kinaseactive site and subsequently promote a conformational change in the kinase domain that dissociates the NLS and transcriptional activation domain (TAD), driving nuclear translocation of ERK5 and stimulation of a known ERK5-regulated promoter (KLF2). Therefore, ERK5i binding causes paradoxical activation of the ERK5 TAD and its downstream targets. Use of ERK5i that activate the ERK5 TAD and ERK5-dependent pathways may not only hinder research into defining the role of ERK5 in cells but may also impede the therapeutic potential of ERK5i. To aid the development of ERK5 inhibitors as potential therapeutics and tool compounds we set out to understand how ERK5i activated ERK5.

Several lines of evidence indicate that ERK5i elicit paradoxical TAD activation by binding directly to the kinase-active site rather than through off-target effects or feedback loops. Multiple feedback loops function in the ERK1/2 pathway (reviewed in Caunt et al. $)^{51}$ and although none have been described to date for the ERK5 pathway they are likely to exist. We therefore set out to address if inhibition of ERK5 by ERK5i repressed a negative feedback loop, leading to activation of a non-inhibitor bound pool of ERK5 that could lead to pathway output. First, we used kinase dead ERK5. Had ERK5 kinase activity been required for paradoxical pathway activation then kinase dead ERK5 would be insensitive to ERK5i. However, both compound 26 or AX15836 'activated' kinase dead ERK5 in the ERK5:MEF2D assay (Fig. 2). In addition, we did not see an increase in phosphorylation of the TEY motif in the ERK5 activation-loop with compound 26 or phosphorylation of the ERK5 substrate MEF2D showing that neither MEK5 nor ERK5 kinase activity was activated in the presence of ERK5i (Supplementary Figs. 2 and 3). Further evidence against a negative feedback loop came from the MEK5 inhibitor, BIX02189. If inhibition of ERK5 blocked a feedback loop to reactivate the pathway then MEK5 inhibition would be expected to do the same; indeed, this is seen with MEK1/2 inhibitors in cells with wild-type BRAF ${ }^{51}$. In fact, BIX02189 fully inhibited ERK5:MEF2D activity; no residual activity or pathway activation was seen (Fig. 2). Taken together, these results argue against a feedback mechanism being responsible for increased output of ERK5 activity in the presence of ERK5i. To rule out any off-target mechanisms we generated ERK5i-insensitive, kinaseactive mutants of ERK5 (Fig. 3). These fell into two classes with attenuated sensitivity to compound 26; mutations that reduced the effect of compound 26 (I115V and D143G) and those that blocked the effect of compound 26 (L189F and G199C) on ERK5: MEF2D transcriptional activity (Fig. 4). These mutations also blocked ERK5 nuclear localisation in response to compound $\mathbf{2 6}$ (Fig. 5). Taken together, these results show that it is the direct binding of the inhibitors (compound 26 or AX15836) to the ERK5 kinase-active site that promoted paradoxical activation.

How does binding of ERK5i to the kinase domain activate ERK5 transcriptional activity? The ERK5 kinase domain and TAD are known to reciprocally repress each other in cells ${ }^{6,11}$. When ERK5 is activated by MEK5 this leads to autophosphorylation of the ERK5 C-terminus, which promotes transcriptional activity ${ }^{11}$. It is known that inhibitor binding can alter the dynamic ensemble of kinase conformations to stabilise a specific conformation ${ }^{52}$. The crystal structure of ERK5 in complex with compound 25 shows that ERK5 is in an 'active' conformation using PKA in complex with ATP and PKI as a reference (Supplementary Fig. 7). Interestingly, comparison of the crystal structures of ERK5 in complex with compound 25 (PDB 4B99) and with ATP and the PB1 domain of MEK5 (PDB 4IC7) (Supplementary Fig. 7) reveals differences in the conformation of the activation-loop and the orientation of the $\alpha \mathrm{C}$-helix that might propagate to changes in the C-terminal portion of the protein (however, both of these regions are also involved in crystal lattice contacts so caution should be exercised in the interpretation of the observed differences). To assess the potential impact of AX15836 binding to ERK5 we conducted molecular dynamic simulations. The binding energy was less favourable for AX15836 compared to compound $\mathbf{2 6}$, however, there was a reduction in the flexibility of the activation-loop most notably at positions 215 and 216-adjacent to the TEY motif at 218-220 (Supplementary Fig. 8). These observations suggest that either the conformational 


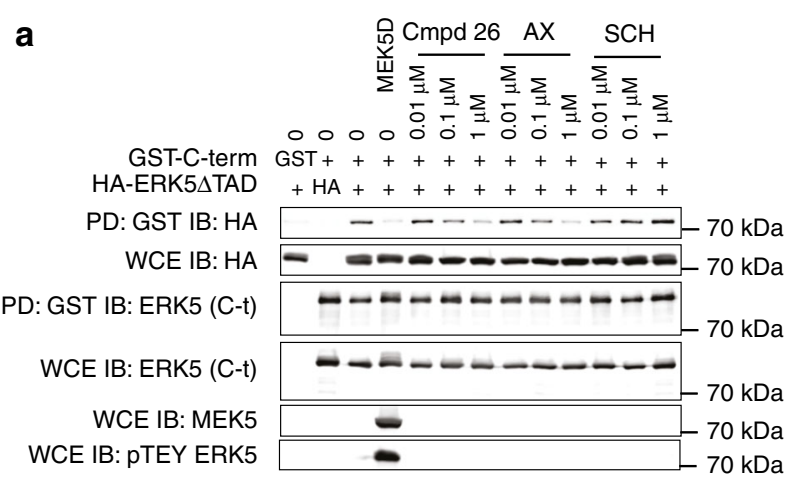

c
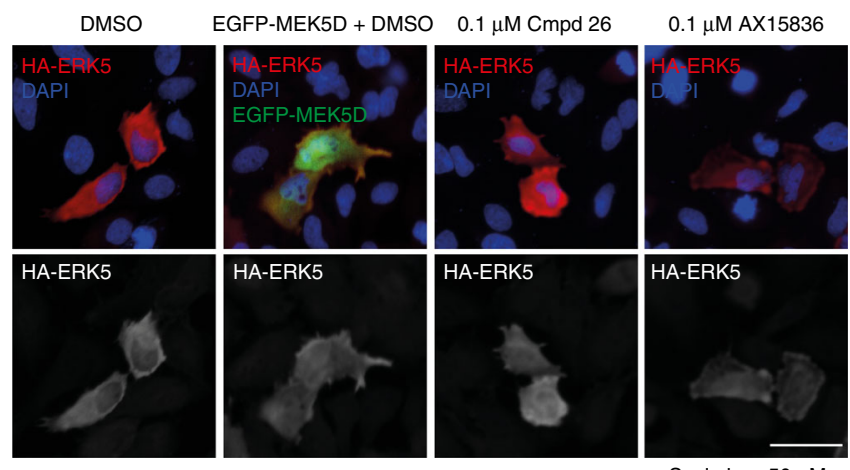

Scale bar: $50 \mu \mathrm{M}$
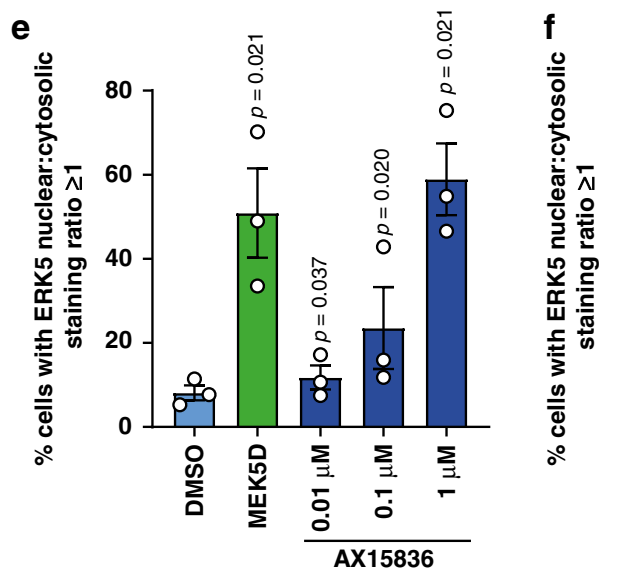

h

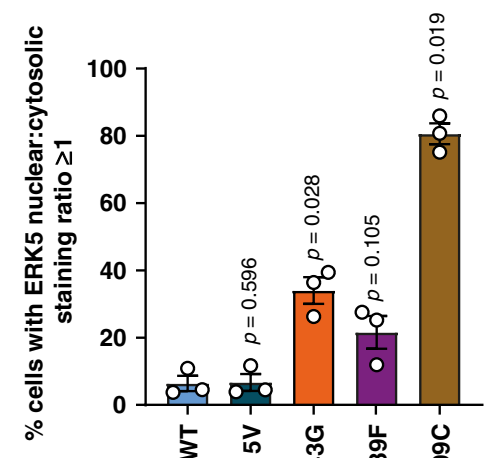

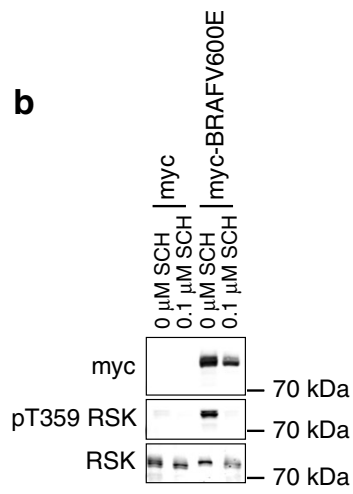

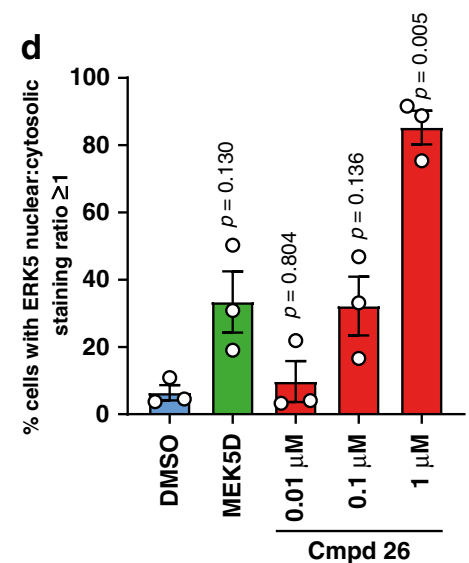

g

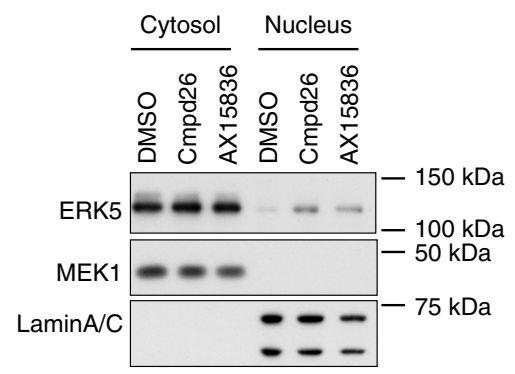

changes in the kinase domain are involved in interactions between the kinase domain and C-terminus, or that the inhibitor directly blocks such interactions. Further molecular analysis is required to characterise these intramolecular interactions to fully validate this model.

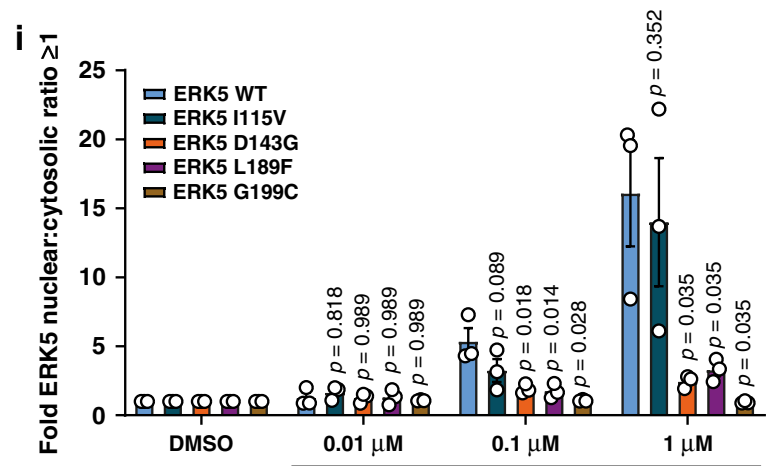

Paradoxical activation of ERK5 is the second major hurdle that ERK5 inhibitor programmes have encountered. The first hurdle emerged from studies with the founding ERK5 inhibitor, XMD892. This compound is tenfold more selective for ERK5 over other kinases tested and phenocopied siRNA knockdown of 
Fig. 5 Compound 26 and AX15836 promote ERK5 nuclear localisation. a HEK293 cells were transfected with GST-ERK5 C-term, HA-ERK5 $\triangle T$ TAD, GST, HA, EGFP-MEK5D or GFP as indicated. Four hours post transfection, cells were treated with compound 26, AX15836 (AX), SCH772984 (SCH) or DMSO as control $(0)$ at the concentrations indicated for $24 \mathrm{~h}$ then the cells were lysed. Lysates were incubated with glutathione sepharose to precipitate GST-ERK5 C-term and measure co-pulldown of HA-ERK5 $\triangle$ TAD. Pulldowns (PD) were immuno-blotted (IB) for GST-ERK5 C-term using ERK5 (C-terminal) antibodies and HA-ERK5 $\triangle T A D$ using HA antibodies. To measure expression, whole-cell extracts (WCE) were blotted using ERK5 (C-terminal), HA and MEK5. Phosphorylation of ERK5 by MEK5D was measured using phospho-ERK5 TEY antibodies. Source data are provided as a Source Data file. $\mathbf{b}$ HEK293 cells were transfected with either myc-tag or myc-BRAFV600E as indicated. Four hours post transfection, cells were treated with $0.1 \mu \mathrm{M} \mathrm{SCH772984} \mathrm{(SCH)}$ or DMSO as control (0). Cells were harvested and immuno-blotted for myc (for expression of myc-BRAFV600E), phospho-T359 RSK and total RSK. A representative image of three independent experiments is shown. Source data are provided as a Source Data file. c HeLa cells were transfected with wildtype HA-ERK5 and either EGFP or EGFP-MEK5D. Twenty-four hours post transfection, cells were treated with compound 26, AX15836 or DMSO as indicated. Thirty minutes after treatment cells were fixed, permeabilised, blocked and stained with anti-HA then donkey-anti-mouse 568 Alexafluor and DAPI. Images were captured by high-content fluorescence microscopy. $\mathbf{d}$ and $\mathbf{e}$ HeLa cells were transfected with wild-type HA-ERK5 and either EGFP or EGFP-MEK5D. Four hours post transfection, cells were treated with compound $\mathbf{2 6}$ (d), AX15836 (e) or DMSO as indicated. Twenty-four hours post transfection, cells were fixed, permeabilised, blocked and stained with anti-HA then donkey-anti-mouse 568 Alexafluor and DAPI. High-content microscopy was used to determine the levels of nuclear and cytosolic HA-ERK5 staining. Results are presented as the \% of transfected cells with nuclear $\geq$ cytosolic HA staining of three independent experiments \pm SEM. Source data are provided as a Source Data file. $\mathbf{f}$ HeLa cells were transfected as in (d) and (e) then treated with compound $\mathbf{2 6}$ or AX15836 for the times indicated. Cells were then treated and processed as in ( $\mathbf{d}$ and $\mathbf{e})$. $\mathbf{g}$ HeLa cells were treated with $0.1 \mu \mathrm{M}$ compound 26, AX15836 or DMSO for 30 min. Cells were harvested and the cytosolic and nuclear fractions were isolated and immuno-blotted for ERK5, MEK1 (cytosolic marker) and lamin A/C (nuclear marker). A representative image of three independent experiments is shown. Source data are provided as a Source Data file. $\mathbf{h}$ HeLa cells were transfected with wild-type HA-ERK5, HA-1115V ERK5, HA-D143G ERK5, HA-L189F ERK5 or HA-G199C ERK5 and EGFP. Twenty-four hours post transfection, cells were treated and processed as in (d). i HeLa cells were transfected with wild-type HA-ERK5, HA-I115V ERK5, HAD143G ERK5, HA-L189F ERK5 or HA-G199C ERK5 and EGFP. Four hours post transfection, cells were treated with compound $\mathbf{2 6}$ or DMSO as indicated. Twenty-four post transfection, cells were treated and processed as in (d).

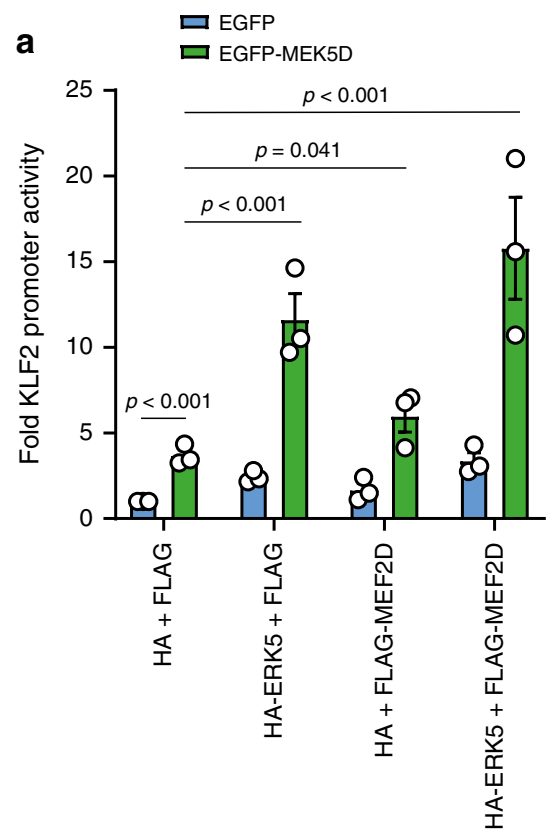

b

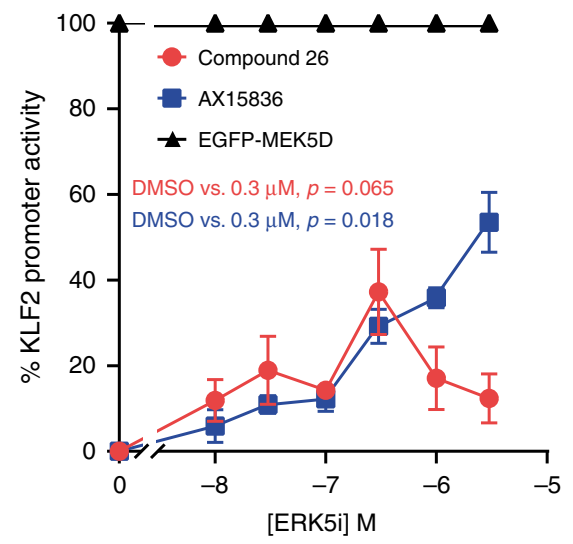

C

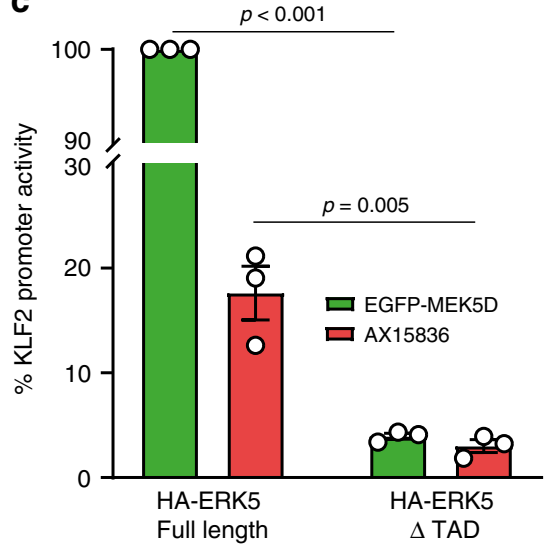

Fig. 6 ERK5i induce expression from the KLF2 promoter and is dependent on ERK5 TAD. a HEK293 cells were transfected with KLF2:LUC and CMV: Renilla together with either FLAG or FLAG-MEF2D and either HA or HA-ERK5 (full length), and either EGFP (control) or EGFP-MEK5D as indicated. Twenty-four hours post transfection, cells were lysed and firefly luciferase activity was measured and normalised to Renilla. The results are presented as the mean of three independent experiments \pm SEM. Source data are provided as a Source Data file. $\mathbf{b}$ HEK293 cells were transfected with KLF2:LUC and CMV:Renilla together with FLAG and HA, with either EGFP or EGFP-MEK5D (to activate endogenous ERK5). Four hours post transfection, cells transfected with KLF2:LUC and CMV:Renilla together with FLAG, HA and EGFP were treated with either compound 26, AX15836 or DMSO (control) at the concentrations indicated. Cells transfected with KLF2:LUC and CMV:Renilla together with FLAG, HA and EGFP-MEK5D were treated with DMSO. Twentyfour hours post transfection, cells were lysed and firefly luciferase activity was measured and normalised to Renilla. The results are presented as \% KLF2 promoter activity where MEK5D-driven (through endogenous ERK5) KLF2 promoter activity is 100\% (mean of three independent experiments \pm SEM). Source data are provided as a Source Data file. c HEK293 cells were transfected with KLF2:LUC and CMV:Renilla, together with FLAG-MEF2D and either HA-ERK5 (full length) or HA-ERK5 $\triangle T A D$, and either EGFP (control) or EGFP-MEK5D. Four hours post transfection cells were treated with either $300 \mathrm{nM}$ AX15836 or DMSO (control). Twenty-four hours post transfection cells were lysed and processed as in $\mathbf{b}$.

ERK5 ${ }^{18,27,33,53-56}$. Subsequently, Lin et al. ${ }^{28}$ developed AX15836, a next generation ERK5i with high selectivity and increased potency for ERK5. However, XMD8-92 and AX15836 were found to diverge in key cellular activities, leading to the suggestion that
XMD8-92 had ERK5-independent off-target effects. Indeed, XMD8-92 was found to bind to BRD4 ${ }^{28}$. However, Lin et al. ${ }^{28}$ also noted a discrepancy between the effects of knockdown of ERK5 and ERK5 inhibition by AX15836; knockdown of ERK5 
prevented IL-6 and IL-8 production from HUVEC and BEAS-2B cells, but AX15836 did not. Our results now provide a possible explanation for why AX15836 was unable to phenocopy ERK5 knockdown; namely because AX15836 paradoxically activates ERK5 transcriptional activity. Further studies are now required to address the role of transcriptional regulation by ERK5 in this and other models.

These observations raise two critical questions moving forward. First, since ERK5 kinase inhibitors can activate the ERK5 TAD, which of these two activities (kinase domain or TAD) is critical for the biological effects of ERK5 in diseases such as cancer and inflammation? In this context it is notable that naturally occurring ERK5 splice variants exist that lack either the C-terminal TAD or the N-terminus, including the kinase $\mathrm{N}$-lobe (such that the kinase will be inactive ${ }^{42,57}$; it will be interesting to see if the embryonic lethality associated with ERK5 knockout is replicated by a $\triangle T A D$ deletion or a catalytically inactive kinase mutant. The second critical question is whether paradoxical activation by ERK5i can be engineered out? The answer to this question is likely to be yes. The relative magnitude of effect of compound $\mathbf{2 6}$ and AX15836 (Fig. 2) suggests there is scope for this effect to be eliminated through the combination of structure-guided drug design and cell-based screening. Using BRAF as an example, success has been seen with the paradox-breaking compounds, PLX7904 and PLX8394. These inhibitors suppress the growth of melanoma cells with $\mathrm{BRAF}^{\mathrm{V} 600 \mathrm{E}}$ but, unlike vemurafenib, do not activate ERK1/2 signalling in cells harbouring mutant KRAS or in cells that have activated tyrosine kinases ${ }^{58}$.

To date, two mechanisms of paradoxical pathway activation have been described for kinase inhibitors. The first is by priming kinases for activation. For example, selective inhibitors of PKB promote its membrane localisation, phosphorylation of its regulatory sites (T308 and S473) by the upstream kinases, PDK1 and mTOR, respectively ${ }^{52}$, and acquisition of a phosphatase-resistant conformation ${ }^{59}$. As a result, when the inhibitor dissociates, PKB is catalytically active and primed for activity ${ }^{50}$. Similar observations have been made for $\mathrm{PKC}^{60}, \mathrm{PKD}^{61}$ and $\mathrm{AMPK}^{62}$. The second way small-molecule kinase inhibitors can paradoxically activate a pathway is by transactivation of non-drug-bound isoforms in homo- or hetero-dimer pairs. The most notable example are the RAF inhibitors such as vemurafenib. This inhibitor was developed to target the $\mathrm{BRAF}^{\mathrm{V} 600 \mathrm{E}}$ mutant and shows potent anti-tumour effects in tumours carrying this mutation. Critically, while vemurafenib can inhibit $\mathrm{BRAF}^{\mathrm{V} 600 \mathrm{E}}$, which signals as a monomer, it can activate wild-type RAF kinases that act as RAS-dependent CRAF-CRAF homodimers or BRAF-CRAF heterodimers. In this case, the drugbound protomer trans-activates the drug-free protomer, leading to pathway activation. Inhibitor binding also promoted membrane localisation of RAF and interaction with RAS-GTP63,64. Similar observations have been made for bosutinib binding to the pseudokinase HER3 ${ }^{65,66}$ to induce an EGFR-dependent proliferative signal ${ }^{67}$. What we observe with inhibitor binding to ERK5 presents a third and entirely new way that kinase inhibitors can cause paradoxical activation of their intended target. Direct binding of the inhibitor to the kinase causes intramolecular changes that cause activation of the target molecule. This is neither dependent on priming phosphorylation and inhibitor dissociation, nor transactivation of a dimer partner to induce phosphorylation of downstream targets. We term this 'direct paradoxical activation by kinase inhibitors'.

In conclusion, we show that ERK5i paradoxically activate ERK5 transcriptional activity by binding to the ERK5 catalytic site and de-repressing the C-terminal TAD function; this represents a third model whereby kinase inhibitors paradoxically activate their intended target. This may explain why the most upto-date ERK5i that lack BRD4 activity do not phenocopy ERK5 small-interfering RNA (siRNA) knockdown. As a result, caution should be exercised when interpreting cellular data using compound 26 (ERK5-IN-1/XMD17-109) or AX15836. Furthermore, paradox-breaking ERK5i should be identified to progress our understanding of ERK5 structure-function, ERK5 biology and to inform ERK5 drug discovery programmes.

\section{Materials and methods}

Materials. Cell culture reagents were purchased from Invitrogen and Sigma. Compounds 25 and 26 (ERK5-IN-1) were originally provided by Professor Nathanael Gray and Dr. Jinhua Wang, Department of Biological Chemistry and Molecular Pharmacology, Harvard Medical School, Boston, USA then latterly compound 26 was purchased from Selleckchem. AX15836 and JQ1 are from Tocris. BIX02189 is from Selleckchem. Anti-HA antibody for immunoblots was provided by the Babraham Institute Monoclonal Facility ${ }^{40,41}$ and anti-HA (12CA5) antibody for immuno-fluorescence ${ }^{68}$ was from Roche/Sigma. Phospho-ERK5 TEY (3371) antibody was from Cell Signaling Technology ${ }^{40,41}$. Phospho-T733 and phospho-S754 ERK5 antibodies were a kind gift from Dr. Atanasio Pandiella, University of Salamanca, Spain ${ }^{16}$. Anti-Flag antibody (M2) is from Sigma ${ }^{69}$. AntiMEK5 antibody (AB3184) is from EMD Millipore ${ }^{22}$, anti-lamin A/C $(636)^{70}$, ERK5 $(\mathrm{C}-7)^{26}$ and myc (9E10) ${ }^{71}$ antibodies are from Santa Cruz and anti-MEK1/2 ${ }^{72}$, phospho-T359 RSK $^{73}$ and total RSK ${ }^{74}$ from Cell Signaling Technology. Secondary antibodies were from BioRad ${ }^{40,41}$.

\section{Methods}

Cell culture. HEK293 and HeLa were cultured in Dulbecco's modified Eagle medium (DMEM) supplemented with $10 \%$ FBS at $37^{\circ} \mathrm{C}$ in an atmosphere of $5 \%$ $\mathrm{CO}_{2}$ and $95 \%$ humidity.

Plasmids. The HA-ERK5 and EGFP-MEK5D constructs have been described previously and were derived from constructs provided by J.D. Lee. GAL4-MEF2D (GAL4 DNA binding domain fused to a.a. 87-428 of rat MEF2D) was from J.C. McDermott, Centre for Research in Biomolecular Interactions, York University, Toronto, Canada; GAL4:LUC reporter from D. Gillespie, University of Glasgow, Glasgow, UK; GST-ERK5 C-term (aa 401-815) was from J. Lizcano, Universitat Autònoma de Barcelona, Spain. A luciferase reporter gene driven by the KLF2 promoter $(-924+14)$ was a kind gift from J. Abe, Department of Cardiology, Division of Internal Medicine, The University of Texas MD Anderson Cancer Centre, Houston, Texas, U.S.A. p3XFlag-MEF2D was a gift from R. Prywes (Addgene plasmid \# 32964).

HA-ERK5 D182A, I115V, D143G, L189F, G199C, S754A, S754D, $\Delta$ TAD, Y741*, L664*, A579*, K506* and P435* were made by site-directed mutagenesis using either QuikChange Site-Directed Mutagenesis kit (Agilent) or Q5 SiteDirected Mutagenesis Kit (New England Biolabs) and verified by ABI automated sequencing.

Luciferase assays. In 96-well tissue culture plates with opaque sides (Thistle Scientific), HEK293 were transfected with pEGFP-MEK5D, pCANHA-ERK5, GAL4-MEF2D, 5XGAL4-Luciferase and CMV-renilla using Lipofectamine 2000 according to the manufacturer's instructions. Compounds 25 and 26, AX15836 and BIX02189 were added at concentrations indicated, $4 \mathrm{~h}$ post transfection. After a further $20 \mathrm{~h}$, cells were harvested and processed for firefly and renilla luciferase activity using the Promega Dual Luciferase Reporter assay according to the manufacturer's instructions.

For KLF2-promoter experiments, HEK293 were transfected with pEGFPMEK5D, pCANHA-ERK5, p3XFlag-MEF2D, luciferase reporter gene driven by the KLF2 promoter $(-924$ to +14$)$ and CMV-Renilla using Lipofectamine 2000 according to the manufacturer's instructions and treated as above.

Antibody conditions. Antibodies and conditions used are given in Table 1.

Immunoblot analysis. For western blotting, $6 \mathrm{~cm}$ dishes (Thermo Scientific) were routinely transfected as described then treated with compound 26, AX15836, SCH772984 or DMSO at the indicated concentrations and for the indicated times prior to harvesting and lysed to prepare whole-cell extracts in $150 \mu \mathrm{l}$ TG lysis buffer (20 mM Tris- $\mathrm{HCl} \mathrm{pH} 7.5,137 \mathrm{mM} \mathrm{NaCl}, 1 \mathrm{mM}$, EGTA, 1\% v/v Triton X-100, 10\% v/v glycerol, $1.5 \mathrm{mM} \mathrm{MgCl} 2,1 \mathrm{mM} \mathrm{Na} \mathrm{VO}_{4}, 1 \mathrm{mM}$ PMSF, $10 \mu \mathrm{g} / \mathrm{ml}$ leupeptin, 10 $\mu \mathrm{g} / \mathrm{ml}$ aprotinin, $50 \mathrm{mM} \mathrm{NaF}$ ). Samples were subjected to sodium dodecyl sulfate-polyacrylamide gel electrophoresis (SDS-PAGE) on $10 \%$ gels, then transferred to PVDF membranes. Membranes were blocked in 5\% milk-TBST and incubated with antibody overnight, shaking at $4{ }^{\circ} \mathrm{C}$. The following day, membranes were washed 3 times with TBS-T, incubated for $30 \mathrm{~min}-1 \mathrm{~h}$ with HRP-secondary antibody and the bands imaged with ECL and film. Films were scanned using an EPSON scanner, and images rotated in Adobe Photoshop Elements and the image cut and pasted into Adobe Illustrator to prepare the figures. Alternatively, blots were incubated with IRDye 680 or 800 anti-mouse or anti-rabbit secondary 
Table 1 Antibodies and conditions used.

\begin{tabular}{lllll} 
Antibody & Supplier & Catalogue number & Dilution/ diluent & Technique \\
\hline Anti-MEK5 & EMD Millipore & AB3184 & $1: 500 / 5 \%$ milk TBS-T & Immuno-blotting \\
Anti-phospho- & Cell Signaling & 3371 & $1: 500 / 5 \%$ BSA TBS-T & Immuno-blotting \\
TEY ERK5 & & & & Immuno-blotting \\
Anti-FLAG M2 & Roche/Sigma & F3165 & $1: 2000 / 5 \%$ milk TBS-T & Im \\
Anti-HA (12CA5) & Sigma & 11583816001 & $5 \mu \mathrm{ml}$ in Antibody Dilution Buffer: (1x & Immuno- \\
& & & PBS/1\% BSA/0.3\% Triton ${ }^{\text {TM }}$ X-100) & fluorescence \\
Anti-HA & Babraham Institute Monoclonal Facility & N/A & $1: 10 / 5 \%$ milk TBS-T & Immuno-blotting \\
Anti-phospho-T733 & Gift from Dr. Atanasio Pandiella, & N/A & $1: 250 / 5 \%$ milk TBS-T & Immuno-blotting \\
& University of Salamanca, Spain. & & & \\
Anti-phospho-S754 & Gift from Dr. Atanasio Pandiella, & N/A & $1: 250 / 5 \%$ milk TBS-T & Immuno-blotting \\
& University of Salamanca, Spain. & & & \\
Anti-myc (9E10) & Santa Cruz & SC-40 & $1: 1000 / 5 \%$ milk TBS-T & Immuno-blotting \\
Anti-phospho- & Cell Signaling & 8753 & $1: 1000 / 5 \%$ milk TBS-T & Immuno-blotting \\
T359 RSK & & & & \\
Anti-RSK & Cell Signaling & 9355 & $1: 1000 / 5 \%$ milk TBS-T & Immuno-blotting \\
Anti-MEK1/2 & Cell Signaling & 9122 & $1: 1000 / 5 \%$ milk TBS-T & Immuno-blotting \\
Anti-Lamin A/C & Santa Cruz & SC-7292 & $1: 500 / 5 \%$ milk TBS-T & Immuno-blotting \\
Anti-ERK5 (C-7) & Santa Cruz & SC-398015 & $1: 1000 / 5 \%$ milk TBS-T & Immuno-blotting
\end{tabular}

antibodies and visualised using an Odyssey CLx Li-COR scanner. All uncropped blots and scans are in the Source Data file.

GST pull-downs. For GST pull-downs, $10 \mathrm{~cm}$ dishes (Thermo Scientific) were routinely transfected as described then used to prepare whole-cell extracts in $500 \mu \mathrm{l}$ TG lysis buffer. Two-hundred microlitres lysate was incubated with $20 \mu \mathrm{lglu}-$ tathione sepharose $4 \mathrm{~B}$ (GE Healthcare) (50\% slurry in PBS) at $4{ }^{\circ} \mathrm{C}$ end-over-end for $2 \mathrm{~h}$. Glutathione-sepharose conjugates were washed two times with TG lysis buffer, spinning at $3000 \mathrm{rcf}$ for $1 \mathrm{~min}$, then subjected to SDS-PAGE and immune blotting using the antibodies indicated.

Nuclear-cytosolic fractionation. HeLa cells were seeded in $10 \mathrm{~cm}$ dishes (Thermo Scientific) and allowed to settle for $24 \mathrm{~h}$. Cells were then treated with $0.1 \mu \mathrm{M}$ compound 26, AX15836 or DMSO control for $30 \mathrm{~min}$. Cells were harvested by trypsinisation and centrifugation and snap frozen. The cell pellets were thawed on ice resuspended in ice cold buffer A (10 mM Hepes $\mathrm{pH} 7.9,1.5 \mathrm{mM} \mathrm{MgCl} 2,10 \mathrm{mM} \mathrm{KCl}$, $0.1 \mathrm{mM}$ EDTA, $1 \mathrm{mM} \mathrm{Na} \mathrm{VO}_{4}, 1 \mathrm{mM}$ PMSF, $10 \mu \mathrm{g} / \mathrm{ml}$ leupeptin, $10 \mu \mathrm{g} / \mathrm{ml}$ aprotinin, $50 \mathrm{mM} \mathrm{NaF}$ ). Cell suspensions were incubated on ice to allow cells to swell. In all, $0.1 \%$ NP-40 was added and mixed by gently pipetting up and down. The cell suspension was then passed through a $25 \mathrm{G}$ needle to disrupt the cell membrane. The homogenate was centrifuged at $800 \mathrm{rcf}$ to pellet the nuclei. The cytosolic fraction was transferred to a fresh tube and centrifuged three times at 10,000 rcf and supernatant removed to pellet any remaining nuclei. $\mathrm{NaCl}$ was added to a final concentration of $137 \mathrm{mM}$ and glycerol to $100 \%$. The nuclear fraction was re-suspended in buffer A and centrifuged at $800 \mathrm{rcf}$ and washed three times with buffer A to remove any residual cytosol. The nuclei pellet was re-suspended in RIPA buffer $(50 \mathrm{mM}$ Tris- $\mathrm{HCl} \mathrm{pH} 8$, $150 \mathrm{mM} \mathrm{NaCl}, 1 \% \mathrm{v} / \mathrm{v}$ Triton X-100, $1 \% \mathrm{w} / \mathrm{v}$ sodium deoxycholate, $0.1 \% \mathrm{w} / \mathrm{v}$ SDS, 1 $\mathrm{mM} \mathrm{Na} \mathrm{VO}_{4}, 1 \mathrm{mM}$ PMSF, $10 \mu \mathrm{g} / \mathrm{ml}$ leupeptin, $10 \mu \mathrm{g} / \mathrm{ml}$ aprotinin, $50 \mathrm{mM} \mathrm{NaF}$ ) supplemented with $2.25 \mathrm{U}$ benzonase $/ \mathrm{ml}$ and incubated at $4^{\circ} \mathrm{C}$ for $1 \mathrm{~h}$ end-over-end and occasionally vortexing. Samples were subjected to SDS-PAGE and immunoblotted with the antibodies indicated.

Mass spectrometry. MEF2D bands were cut from Coomassie-stained gels, reduced, carbamidomethylated and digested overnight at $30^{\circ} \mathrm{C}$ in $25 \mathrm{mM}$ ammonium bicarbonate containing $10 \mathrm{ng} / \mathrm{ml}$ of protease (modified trypsin (Promega) or elastase (Promega)). Phosphopeptides were enriched using titanium dioxide beads (Titansphere, GL Sciences) and glycolic acid as competitor. Enriched phosphopeptides, or unfractionated digests, were analysed by nano liquid chromatography-mass spectrometry (LC-MS/MS) on a QExactive Plus mass spectrometer (Thermo Scientific) fitted with a nanoelectrospray ion-source (Proxeon). Peptides were separated on a reversed-phase column $(0.075 \times 150 \mathrm{~mm}$; ReproSil-Pur 120 C18-AQ $3 \mathrm{~mm}$ ) using an acetonitrile gradient (5-40\% in $30 \mathrm{~min}$ at $300 \mathrm{nl} / \mathrm{min}$ ) containing $0.1 \%$ formic acid. Full scan data were acquired over the $\mathrm{m} / \mathrm{z}$ range $350-1800$ at a nominal resolution setting of 70,000 , followed by up to 10 higher-energy collisional dissociation (HCD) spectra at $27 \%$ relative collision energy. Analyses of the digests with targeted MS/MS of the identified phosphopeptides were done similarly, but with HCD fragmentation at $35 \%$ relative collision energy. Mass spectral data were searched against the human entries of the Uniprot 15.14 database using Mascot software (Matrix Science) and all putative phosphopeptide MS/MS spectra were manually validated. Apparent stoichiometries of phosphorylation were calculated from the peak areas of the extracted ion chromatograms of the phospho- and corresponding nonphosphopeptides in LCMS runs of unfractionated digests as in ref. ${ }^{40}$.

(Phospho)peptides were identified from mass spectrometry data using Peaks Studio V7 (Bioinformatics Solutions Inc) and manually validated, while quantitative analyses were done with the aid of Skyline Software (MacCoss Lab, Washington University).

Molecular dynamics simulations. The structure of ERK $5^{37}$ co-crystallised with the specific inhibitor compound 25 (PDB: 4B99) was used as a starting model for molecular dynamics simulation. All solvent molecules were removed, and compound 25 was extracted from the model. Missing loops were built with MODELLER (v9.19) ${ }^{75,76}$ via UCSF Chimera ${ }^{77}$. Mutants were modelled in UCSF Chimera. Compound 25 was modified in Avogadro (v.1.9 $)^{78}$ to create compound 26 using the published structure as a ref. 37, changing the R1 group from a methoxy to ethoxy moiety and adding hydrogen atoms. The ligand was then parameterised using Gasteiger point charges in the AMBER ff99s $\mathrm{s}^{79}$ force field for simulation using Antechamber ${ }^{80}$, and prepared for GROMACS using ACPYPE ${ }^{81}$. The complexes were then prepared in GROMACS ${ }^{82}$, parameterised in ff99sb and neutralised with sodium and chloride ions to a final concentration of $0.1 \mathrm{M}$, prior to being subject to minimisation and equilibration. Briefly, a steepest descent gradient of maximum 5000 steps was used to minimise the complexes, which converged on an $F_{\max }<1000 \mathrm{~kJ} / \mathrm{mol} / \mathrm{nm}$ in $<2000$ steps. The complexes were then subject to 200 ps equilibration in the NvT ensemble using a 2 fs timestep and NoseHoover temperature coupling at $300 \mathrm{~K}$; all atoms were subject to position restraints. Subsequent equilibration in the NpT ensemble was also conducted under position restraints at a $2 \mathrm{fs}$ timestep, with Parinello-Rahman barostat at 1 bar. Finally, position restraints were released and three parallel production MD simulations were conducted for a $10 \mathrm{~ns}$.

The g_mmpbs ${ }^{83}$ package was used to calculate binding energies between ligand and protein. The final 2 ns of converged simulation time was used for each of the simulations, totalling 201 snapshots from 8-10 ns. The binding energies were converted from $\mathrm{kJ} / \mathrm{mol}$ to the more commonly used $\mathrm{kcal} / \mathrm{mol}$ and averaged.

To model AX15836 binding to ERK5, the method outlined above for compound 26 was used and run for $100 \mathrm{~ns}$ and the root mean squared fluctuation (RMSF) of each residue was calculated for the period $10-100 \mathrm{~ns}$.

High-content microscopy: equipment and settings. HeLa cells were seeded in Cell CarrierTM-96 plates (Perkin Elmer) then transfected with pCANHA-ERK5 and pEGFP-MEK5D using Extreme-Gene 9 (Sigma) as indicated. Cells were treated with compound $\mathbf{2 6}$ or DMSO at the concentrations and for the indicated times prior to fixing with $4 \%$ paraformaldehyde for $30 \mathrm{~min}$ at $25^{\circ} \mathrm{C}$. The cells were then blocked with Blocking Buffer (1x PBS/5\% goat serum/0.3\% Triton ${ }^{\mathrm{mm}} \mathrm{X}-100$ ). Slides were then incubated with anti-HA (12CA5) 1:100 in Antibody Dilution Buffer: (1X PBS/1\% BSA $/ 0.3 \%$ Triton $^{\text {mt }} \mathrm{X}-100$ ) overnight at $4{ }^{\circ} \mathrm{C}$. Slides were washed 3 times with PBS for 5 min per wash then incubated with 1:1000 donkeyanti-mouse 568 Alexa Fluor (Invitrogen) and $1 \mu \mathrm{g} / \mathrm{ml}$ DAPI. Slides were then washed three times for $5 \mathrm{~min}$ per wash and left in PBS for imaging.

Cells were imaged using In Cell 6000 at room temperature, with 20x objective lens acquiring GFP, DAPI and dsRed using the InCell software settings. Single section images of xy planes with pixel dimensions $325 \times 325 \mathrm{~nm}$ and 16 bit image $(2048 \times$ 2048 pixels). The fluorochromes used were 568 Alexa Fluor (Laser: 568 nm, Filter: 
Texas Red, Excitation max: $578 \mathrm{~nm}$, and Emission max: $603 \mathrm{~nm}$ ), DAPI (Laser: 405 nm, Filter: DAPI, Excitation max: $350 \mathrm{~nm}$, and Emission max: $470 \mathrm{~nm}$ ), and GFP (Laser: $488 \mathrm{~nm}$, Filter: FITC, Excitation max: $488 \mathrm{~nm}$, and Emission max: $510 \mathrm{~nm}$ ). Results were analysed using In Cell Analyser Workstation 3.7.2 to determine the level of nuclear and cytosolic ERK5. In the Assay Parameters for Nuclei and Cells, Segmentation of nuclei was set to Top-Hat with a minimum area of $50 \mu \mathrm{m}^{2}$ and Segmentation of cells to Collar with a radius of $2 \mu \mathrm{m}$. The Assay Measures were Nuclei Area, Nucl/(FormFactor), Nuclei Intensity; Reference, Nuc/Cell Intensity, Nuc Intensity, Cell Intensity; Cell tracking, Label, Linked Track ID; and Filters, threshold, Nucl/(Form Factor), Nuclei. The Cell-by-Cell data was exported to Microsoft Excel as a.csv file. Transfected cells were identified by having a reading $>$ than $2 x$ background intensity. The \% of cells with higher nuclear ERK5 staining to cytosolic ERK5 staining was calculated for each experimental well.

Representative.tiff images we merged in ImageJ and the scale bar added. The images were converted to RGB colour, or the red channel displayed as greyscale alone. The brightness/contrast minimum and maximum was fixed to the same values for each image.

Statistical analysis. Depending on the scientific question, in particular the number of conditions compared, and the experimental design, independent or paired $t$-tests or one-way or repeated measured analysis of variances (ANOVAs) were run to establish statistical significance $(p<0.05)$. ANOVAs were followed by post-hoc tests with Holm-Sidak correction for multiple comparisons. When the experiments were comparing curves, area under the curves were used for the statistical analyses. Where appropriate, to show significance or not, the $p$-values are displayed on the graphs.

Reporting summary. Further information on research design is available in the Nature Research Reporting Summary linked to this article.

\section{Data availability}

The data sets analysed during the current study are available in the following repositories: coordinates for the structures of the ERK5-Cpd 25, ERK5-ATP-MEK5 PB1 domain, and PKA-ATP-PKI complexes were retrieved from the Protein Databank in Europe (PDBe) at https://www.ebi.ac.uk/pdbe/ using the accession codes 4B99, 4IC7 and 4WB5, respectively; protein sequences for human AURKA, AURKB, DCLK1, DLCK2, DCLK3, ERK1, ERK2, ERK3, ERK4, ERK5, ERK6, ERK7, ERK8, LRRK2, PLK1, PLK2, PLK3, PLK4, RIPK1, RIPK2 and RIPK5, and for human, mouse and rat MEF2D were retrieved from the UniProt knowledge Base at https://www.uniprot.org/ using the accession codes O14965, Q96GD4, O15075, Q8N568, Q9C098, P27361, P28482, Q16659, P31152, Q13164, P53778, Q8TD08, Q5S007, P53350, Q9NYY3, Q9H4B4, O00444, Q13546, O43353, Q6XUX3, Q14814, Q63943 and O89038, respectively. The source data underlying Figs. $2 \mathrm{a}-\mathrm{h}, 3 \mathrm{~d}-\mathrm{k}, 4 \mathrm{a}-\mathrm{k}, 5 \mathrm{a}, \mathrm{b}, \mathrm{d}-\mathrm{i}$ and $6 \mathrm{a}-\mathrm{c}$, and Supplementary Figs. S1B-D, S2A-F, S3C, S4C, D, S5, S6, S8A-C are provided as a Source Data file. All the other data supporting the findings of this study are available within the article and its supplementary information files and from the corresponding author on reasonable request.

\section{Code availability}

All code used to generate and analyse the Molecular Dynamic Simulations can be accessed here https://osf.io/thfx3/

Received: 20 September 2018; Accepted: 11 February 2020; Published online: 13 March 2020

\section{References}

1. Lee, J. D., Ulevitch, R. J. \& Han, J. Primary structure of BMK1: a new mammalian map kinase. Biochem. Biophys. Res. Commun. 213, 715-724 (1995).

2. Zhou, G., Bao, Z. Q. \& Dixon, J. E. Components of a new human protein kinase signal transduction pathway. J. Biol. Chem. 270, 12665-12669 (1995).

3. Johnson, G. L. ERK1/ERK2 MAPK pathway. (Connections Map in the Database of Cell Signaling, as seen 27 February 2014), http://stke.sciencemag. org/cgi/cm/stkecm;CMP_10705. Sci. Signal. (2005).

4. Johnson, G. L. JNK MAPK Pathway. (Connections Map in the Database of Cell Signaling, as seen 27 February 2014) http://stke.sciencemag.org/cgi/cm/ stkecm;CMP_10827. Sci. Signal. (2003).

5. Johnson, G. L. p38 MAPK Pathway. (Connections Map in the Database of Cell Signaling, as seen 27 February 2014), http://stke.sciencemag.org/cgi/cm/ stkecm;CMP_10958. Sci. Signal.(2008).

6. Kasler, H. G., Victoria, J., Duramad, O. \& Winoto, A. ERK5 is a novel type of mitogen-activated protein kinase containing a transcriptional activation domain. Mol. Cell. Biol. 20, 8382-8389 (2000).
7. Kato, Y. et al. BMK1/ERK5 regulates serum-induced early gene expression through transcription factor MEF2C. EMBO J. 16, 7054-7066 (1997).

8. Wilhelmsen, K., Mesa, K. R., Lucero, J., Xu, F. \& Hellman, J. ERK5 protein promotes, whereas MEK1 protein differentially regulates, the Toll-like receptor 2 protein-dependent activation of human endothelial cells and monocytes. J. Biol. Chem. 287, 26478-26494 (2012).

9. Abe, J., Kusuhara, M., Ulevitch, R. J., Berk, B. C. \& Lee, J. D. Big mitogenactivated protein kinase 1 (BMK1) is a redox-sensitive kinase. J. Biol. Chem. 271, 16586-16590 (1996).

10. Mody, N., Campbell, D. G., Morrice, N., Peggie, M. \& Cohen, P. An analysis of the phosphorylation and activation of extracellular-signal-regulated protein kinase 5 (ERK5) by mitogen-activated protein kinase kinase 5 (MKK5) in vitro. Biochemical J. 372, 567-575 (2003).

11. Morimoto, H., Kondoh, K., Nishimoto, S., Terasawa, K. \& Nishida, E. Activation of a C-terminal transcriptional activation domain of ERK5 by autophosphorylation. J. Biol. Chem. 282, 35449-35456 (2007).

12. Buschbeck, M. \& Ullrich, A. The unique C-terminal tail of the mitogenactivated protein kinase ERK5 regulates its activation and nuclear shuttling. J. Biol. Chem. 280, 2659-2667 (2005).

13. Kato, Y., Chao, T. H., Hayashi, M., Tapping, R. I. \& Lee, J. D. Role of BMK1 in regulation of growth factor-induced cellular responses. Immunologic Res. 21, 233-237 (2000).

14. Kato, Y. et al. Big mitogen-activated kinase regulates multiple members of the MEF2 protein family. J. Biol. Chem. 275, 18534-18540 (2000).

15. Honda, T. et al. Phosphorylation of ERK5 on Thr732 is associated with ERK5 nuclear localization and ERK5-dependent transcription. PLOS ONE 10, e0117914 (2015).

16. Diaz-Rodriguez, E. \& Pandiella, A. Multisite phosphorylation of Erk5 in mitosis. J. Cell Sci. 123, 3146-3156 (2010).

17. Inesta-Vaquera, F. A. et al. Alternative ERK5 regulation by phosphorylation during the cell cycle. Cell Signal. 22, 1829-1837 (2010).

18. Wilhelmsen, K. et al. Extracellular signal-regulated kinase 5 promotes acute cellular and systemic inflammation. Sci. Signal. 8, ra86 (2015).

19. Finegan, K. G. et al. ERK5 is a critical mediator of inflammation-driven cancer. Cancer Res. 75, 742-753 (2015)

20. Giurisato, E. et al. Myeloid ERK5 deficiency suppresses tumor growth by blocking protumor macrophage polarization via STAT3 inhibition. Proc. Natl Acad. Sci. USA 115, E2801-E2810 (2018).

21. Hoang, V. T. et al. Oncogenic signaling of MEK5-ERK5. Cancer Lett. 392, 51-59 (2017)

22. Lochhead, P. A., Gilley, R. \& Cook, S. J. ERK5 and its role in tumour development. Biochemical Soc. Trans. 40, 251-256 (2012).

23. Simoes, A. E., Rodrigues, C. M. \& Borralho, P. M. The MEK5/ERK5 signalling pathway in cancer: a promising novel therapeutic target. Drug Discov. Today 21, 1654-1663 (2016).

24. Zen, K. et al. ERK5 is a target for gene amplification at $17 \mathrm{p} 11$ and promotes cell growth in hepatocellular carcinoma by regulating mitotic entry. Genes Chromosomes Cancer 48, 109-120 (2009).

25. Lochhead, P. A. et al. Tumor cells with KRAS or BRAF mutations or ERK5/ MAPK7 amplification are not addicted to ERK5 activity for cell proliferation. Cell Cycle 15, 506-518 (2016).

26. Tusa, I. et al. ERK5 is activated by oncogenic BRAF and promotes melanoma growth. Oncogene 37, 2601-2614 (2018).

27. Song, C. et al. Targeting BMK1 Impairs the Drug Resistance to Combined Inhibition of BRAF and MEK1/2 in Melanoma. Sci. Rep. 7, 46244 (2017).

28. Lin, E. C. et al. ERK5 kinase activity is dispensable for cellular immune response and proliferation. Proc. Natl Acad. Sci. USA 113, 11865-11870 (2016).

29. Lemos, C. et al. Discovery and profiling of a highly potent and selective ERK5 inhibitor: BAY-885. In Proc. Am. Assoc. Cancer Res. Annu. Meeting. Apr 14-18 (Chicago, IL, 2018).

30. Tatake, R. J. et al. Identification of pharmacological inhibitors of the MEK5/ ERK5 pathway. Biochem. Biophys. Res. Commun. 377, 120-125 (2008).

31. Chen, H. et al. Discovery of a novel allosteric inhibitor-binding site in ERK5: comparison with the canonical kinase hinge ATP-binding site. Acta Crystallogr. Sect. D., Struct. Biol. 72, 682-693 (2016).

32. Deng, X. et al. Discovery of a benzo[e]pyrimido-[5,4-b][1,4]diazepin-6(11H) one as a potent and selective inhibitor of Big MAP kinase 1. ACS Medicinal Chem. Lett. 2, 195-200 (2011).

33. Yang, Q. et al. Pharmacological inhibition of BMK1 suppresses tumor growth through promyelocytic leukemia protein. Cancer Cell 18, 258-267 (2010).

34. Patricelli, M. P. et al. In situ kinase profiling reveals functionally relevant properties of native kinases. Chem. Biol. 18, 699-710 (2011).

35. Patricelli, M. P. et al. Functional interrogation of the kinome using nucleotide acyl phosphates. Biochemistry 46, 350-358 (2007).

36. Deng, X. et al. Structural determinants for ERK5 (MAPK7) and leucine rich repeat kinase 2 activities of benzo[e]pyrimido-[5,4-b]diazepine-6(11H)-ones. Eur. J. Medicinal Chem. 70, 758-767 (2013). 
37. Elkins, J. M. et al. X-ray crystal structure of ERK5 (MAPK7) in complex with a specific inhibitor. J. Medicinal Chem. 56, 4413-4421 (2013).

38. Myers, S. M. et al. Identification of a novel orally bioavailable ERK5 inhibitor with selectivity over p38alpha and BRD4. Eur. J. Medicinal Chem. 178, 530-543 (2019).

39. Yang, C. C., Ornatsky, O. I., McDermott, J. C., Cruz, T. F. \& Prody, C. A. Interaction of myocyte enhancer factor 2 (MEF2) with a mitogenactivated protein kinase, ERK5/BMK1. Nucleic Acids Res. 26, 4771-4777 (1998).

40. Gilley, R. et al. CDK1, not ERK1/2 or ERK5, is required for mitotic phosphorylation of BIMEL. Cell Signal. 24, 170-180 (2012)

41. Gilley, R., March, H. N. \& Cook, S. J. ERK1/2, but not ERK5, is necessary and sufficient for phosphorylation and activation of c-Fos. Cell Signal. 21, 969-977 (2009).

42. McCaw, B. J. et al. Identification and characterization of mErk5-T, a novel Erk5/Bmk1 splice variant. Gene 345, 183-190 (2005).

43. Notredame, C., Higgins, D. G. \& Heringa, J. T-Coffee: A novel method for fast and accurate multiple sequence alignment. J. Mol. Biol. 302, 205-217 (2000).

44. Morris, E. J. et al. Discovery of a novel ERK inhibitor with activity in models of acquired resistance to BRAF and MEK inhibitors. Cancer Discov. 3, 742-750 (2013).

45. Wu, P., Nielsen, T. E. \& Clausen, M. H. FDA-approved small-molecule kinase inhibitors. Trends Pharmacol. Sci. 36, 422-439 (2015).

46. Gorre, M. E. et al. Clinical resistance to STI-571 cancer therapy caused by BCR-ABL gene mutation or amplification. Science 293, 876-880 (2001).

47. Little, A. S. et al. Amplification of the driving oncogene, KRAS or BRAF, underpins acquired resistance to MEK1/2 inhibitors in colorectal cancer cells. Sci. Signal. 4, ra17 (2011).

48. Nazarian, R. et al. Melanomas acquire resistance to B-RAF(V600E) inhibition by RTK or N-RAS upregulation. Nature 468, 973-977 (2010).

49. O’Reilly, K. E. et al. mTOR inhibition induces upstream receptor tyrosine kinase signaling and activates Akt. Cancer Res. 66, 1500-1508 (2006).

50. Okuzumi, T. et al. Inhibitor hijacking of Akt activation. Nat. Chem. Biol. 5, 484-493 (2009).

51. Caunt, C. J., Sale, M. J., Smith, P. D. \& Cook, S. J. MEK1 and MEK2 inhibitors and cancer therapy: the long and winding road. Nat. Rev. Cancer 15, 577-592 (2015).

52. Tong, M. \& Seeliger, M. A. Targeting conformational plasticity of protein kinases. ACS Chem. Biol. 10, 190-200 (2015).

53. Sun, X. et al. Benzidine Induces Epithelial-Mesenchymal Transition of Human Bladder Cancer Cells through Activation of ERK5 Pathway. Molecules Cells 41, 188-197 (2018).

54. Rovida, E. et al. The mitogen-activated protein kinase ERK5 regulates the development and growth of hepatocellular carcinoma. Gut 64, 1454-1465 (2015).

55. Bera, A. et al. A positive feedback loop involving Erk5 and Akt turns on mesangial cell proliferation in response to PDGF. Am. J. Physiol. Cell Physiol. 306, C1089-C1100 (2014).

56. Wang, X., Pesakhov, S., Harrison, J. S., Danilenko, M. \& Studzinski, G. P. ERK5 pathway regulates transcription factors important for monocytic differentiation of human myeloid leukemia cells. J. Cell. Physiol. 229, 856-867 (2014).

57. Yan, C., Luo, H., Lee, J. D., Abe, J. \& Berk, B. C. Molecular cloning of mouse ERK5/BMK1 splice variants and characterization of ERK5 functional domains. J. Biol. Chem. 276, 10870-10878 (2001).

58. Zhang, C. et al. RAF inhibitors that evade paradoxical MAPK pathway activation. Nature 526, 583-586 (2015).

59. Chan, T. O. et al. Resistance of Akt kinases to dephosphorylation through ATP-dependent conformational plasticity. Proc. Natl Acad. Sci. USA 108, E1120-E1127 (2011)

60. Cameron, A. J., Escribano, C., Saurin, A. T., Kostelecky, B. \& Parker, P. J. PKC maturation is promoted by nucleotide pocket occupation independently of intrinsic kinase activity. Nat. Struct. Mol. Biol. 16, 624-630 (2009).

61. Kunkel, M. T. \& Newton, A. C. Protein kinase d inhibitors uncouple phosphorylation from activity by promoting agonist-dependent activation loop phosphorylation. Chem. Biol. 22, 98-106 (2015).

62. Ross, F. A. et al. Mechanisms of Paradoxical Activation of AMPK by the Kinase Inhibitors SU6656 and Sorafenib. Cell Chem. Biol. 24, 813-824 e814 (2017).

63. Poulikakos, P. I., Zhang, C., Bollag, G., Shokat, K. M. \& Rosen, N. RAF inhibitors transactivate RAF dimers and ERK signalling in cells with wild-type BRAF. Nature 464, 427-430 (2010).

64. Hatzivassiliou, G. et al. RAF inhibitors prime wild-type RAF to activate the MAPK pathway and enhance growth. Nature 464, 431-435 (2010).

65. Davis, M. I. et al. Comprehensive analysis of kinase inhibitor selectivity. Nat. Biotechnol. 29, 1046-1051 (2011).

66. Levinson, N. M. \& Boxer, S. G. A conserved water-mediated hydrogen bond network defines bosutinib's kinase selectivity. Nat. Chem. Biol. 10, 127-132 (2014).
67. Claus, J. et al. Inhibitor-induced HER2-HER3 heterodimerisation promotes proliferation through a novel dimer interface. eLife 7, pii: e32271 (2018).

68. Kumar, G. R., Shum, L. \& Glaunsinger, B. A. Importin alpha-mediated nuclear import of cytoplasmic poly(A) binding protein occurs as a direct consequence of cytoplasmic mRNA depletion. Mol. Cell. Biol. 31, 3113-3125 (2011).

69. Croft, L. V. et al. hSSB1 associates with and promotes stability of the BLM helicase. BMC Mol. Biol. 18, 13 (2017).

70. Zhang, L., Yang, Z., Huang, W. \& Wu, J. H19 potentiates let-7 family expression through reducing PTBP1 binding to their precursors in cholestasis. Cell Death Dis. 10, 168 (2019).

71. Watanabe, S. et al. Zinc regulates ERp44-dependent protein quality control in the early secretory pathway. Nat. Commun. 10, 603 (2019).

72. Frischknecht, L. et al. BRAF inhibition sensitizes melanoma cells to alphaamanitin via decreased RNA polymerase II assembly. Sci. Rep. 9, 7779 (2019).

73. Zhang, L. et al. FLOT1 promotes tumor development, induces epithelialmesenchymal transition, and modulates the cell cycle by regulating the Erk/ Akt signaling pathway in lung adenocarcinoma. Thorac. Cancer 10, 909-917 (2019).

74. Haghighi, F. et al. bFGF-mediated pluripotency maintenance in human induced pluripotent stem cells is associated with NRAS-MAPK signaling. Cell Commun. Signal. 16, 96 (2018).

75. Webb, B. \& Sali, A. Protein Structure Modeling with MODELLER. Methods Mol. Biol. 1654, 39-54 (2017).

76. Sali, A. \& Blundell, T. L. Comparative protein modelling by satisfaction of spatial restraints. J. Mol. Biol. 234, 779-815 (1993).

77. Pettersen, E. F. et al. UCSF Chimera-a visualization system for exploratory research and analysis. J. Comput Chem. 25, 1605-1612 (2004).

78. Hanwell, M. D. et al. Avogadro: an advanced semantic chemical editor, visualization, and analysis platform. J. Cheminform. 4, 17 (2012).

79. Hornak, V. et al. Comparison of multiple Amber force fields and development of improved protein backbone parameters. Proteins 65, 712-725 (2006).

80. Wang, J., Wang, W., Kollman, P. A. \& Case, D. A. Automatic atom type and bond type perception in molecular mechanical calculations. J. Mol. Graph Model 25, 247-260 (2006)

81. Sousa da Silva, A. W. \& Vranken, W. F. ACPYPE - AnteChamber PYthon Parser interfacE. BMC Res. Notes 5, 367 (2012).

82. Van Der Spoel, D. et al. GROMACS: fast, flexible, and free. J. Comput. Chem. 26, 1701-1718 (2005).

83. Kumari, R., Kumar, R., Open Source Drug Discovery C \& Lynn, A. g_mmpbsa-a GROMACS tool for high-throughput MM-PBSA calculations. J. Chem. Inf. Model 54, 1951-1962 (2014).

84. McNicholas, S., Potterton, E., Wilson, K. S. \& Noble, M. E. Presenting your structures: the CCP4mg molecular-graphics software. Acta Crystallogr. Sect. D., Biol. Crystallogr. 67, 386-394 (2011).

85. The UniProt C. UniProt: the universal protein knowledgebase. Nucleic Acids Res. 45, D158-D169 (2017).

86. Sievers, F. et al. Fast, scalable generation of high-quality protein multiple sequence alignments using Clustal Omega. Mol. Syst. Biol. 7, 539 (2011).

87. Waterhouse, A. M., Procter, J. B., Martin, D. M., Clamp, M. \& Barton, G. J. Jalview Version 2-a multiple sequence alignment editor and analysis workbench. Bioinformatics 25, 1189-1191 (2009).

\section{Acknowledgements}

We would like to thank members of the Cook Lab for their support and encouragement throughout this study. We thank Hanneke Okkenhaug from the Babraham Institute Science Services (Imaging) for help with high-content imaging and Anne SegondsPichon for statistical analysis. We are also grateful to Tim Hammonds (Cancer Research UK Therapeutic Discovery Laboratories) and Markus Muellner (PhoreMost Ltd.) for useful discussions and encouragement. This study was funded by BBSRC project grant BB/N015886/1 (S.J.C. \& P.A.L), an MRC-DPFS award (MR/K007580/1) and Institute Strategic Programme Grants BB/J004456/1 and BB/P013384/1 from BBSRC (S.J.C.). Work in Martin Noble's laboratory was supported by CRUK (Grant CRUK-A21421).

\section{Author contributions}

P.A.L. made the initial observation. P.A.L. and S.J.C. conceived the study with inputs from J.A.T and M.E.M.N. N.J.W. and N.S.G. developed and synthesised compounds 25 and 26. P.A.L., A.M.K., M.A.C. and V.P.J. performed the experiments. J.A.T. performed structure analysis and sequence alignments. N.J.T. performed molecular dynamics simulations. D.O. performed mass spectrometry. P.A.L., J.A.T., N.J.T. and S.J.C. wrote the manuscript with inputs from all authors.

\section{Competing interests}

N.S.G. is a founder, science advisory board member (SAB) and equity holder in Gatekeeper, Syros, Petra, C4 and Soltego. The remaining authors declare no competing interests. 


\section{Additional information}

Supplementary information is available for this paper at https://doi.org/10.1038/s41467020-15031-3

Correspondence and requests for materials should be addressed to P.A.L. or S.J.C.

Peer review information Nature Communications thanks Paul Shapiro and the other anonymous, reviewer(s) for their contribution to the peer review of this work.

Reprints and permission information is available at http://www.nature.com/reprints

Publisher's note Springer Nature remains neutral with regard to jurisdictional claims in published maps and institutional affiliations. (c) Open Access This article is licensed under a Creative Commons Attribution 4.0 International License, which permits use, sharing, adaptation, distribution and reproduction in any medium or format, as long as you give appropriate credit to the original author(s) and the source, provide a link to the Creative Commons license, and indicate if changes were made. The images or other third party material in this article are included in the article's Creative Commons license, unless indicated otherwise in a credit line to the material. If material is not included in the article's Creative Commons license and your intended use is not permitted by statutory regulation or exceeds the permitted use, you will need to obtain permission directly from the copyright holder. To view a copy of this license, visit http://creativecommons.org/ licenses/by/4.0/.

(C) The Author(s) 2020 